\title{
What Do Interindustry Wage Premiums Represent?
}

\author{
Paulo RA Loureiro ${ }^{1}$, Jorge Arbache ${ }^{1}$, Antônio Nascimento Júnior ${ }^{2}$, Roberto Ellery Júnior ${ }^{1} \&$ José C. C. O. Neto $^{2}$ \\ ${ }^{1}$ Department of Economics-University of Brasília, Distrito Federal, Brazil \\ ${ }^{2}$ Department of Business Administration-University of Brasília, Distrito Federal, Brasil \\ Correspondence: Paulo RA Loureiro, Department of Economics, University of Brasília, Distrito Federal, \\ Campus Darcy Ribeiro - Asa Norte CEP: 70910-900 - Brasília. Tel: 55-61-3107-0744. E-mail: \\ pauloloureiro@unb.br
}

Received: April 8, 2021

Accepted: June 28, 2021

Online Published: June 30, 2021

doi:10.5539/ijef.v13n7p105

URL: https://doi.org/10.5539/ijef.v13n7p105

\begin{abstract}
The aim of this paper is to investigate wage structure in Brazil between 2002 and 2015 based on National Household Surveys (PNADs). Given the nature and simultaneity of economic changes in the assessed period, as well as their potential impacts on the labor market, it is reasonable to assume that, despite the stylized fact that wage structure is quite stable, there have been non-negligible changes in the structure of relative wages. First, the labor market has become highly segmented and complex. Second, changes in wage structure have been observed. Relative wages in some sectors have increased, whereas relative wages in other sectors have decreased, suggesting a tendency towards resource allocation to sectors. There are remarkable potential impacts on income and on labor. Sectors that stand out include petroleum and alcohol, mineral extraction, and civil construction. However, if indirect effects are taken into account, via value chains, the petroleum and civil construction sectors have a major contribution. Fund transfers to these sectors would increase good-quality jobs, productivity, and economic value added. Aside from these changes, the international economy has been put through profound transformation (e.g., financial crisis, changes in relative prices), which has affected the sectors unevenly.
\end{abstract}

Keywords: wage determination, interindustry wage differential, segmentation, competitiveness, Brazil

JEL Classification: JEL codes: J31.

\section{Introduction}

Years 2002 to 2015 were important for the Brazilian labor market. The average effective real earnings of people in formal work increased by $189.7 \%$ between 2002 (R639.3) and2015(R 1,852.2) according to the National Household Survey (PNAD), which was way above the real GDP per capita growth (34\%), and labor productivity, which practically remained unchanged. Open unemployment went from $9.2 \%$ in 2002 to $8.5 \%$ or $9.7 \%$ in 2015 . The informal sector shrank, and was, for the first time, smaller than the formal sector. Labor force participation rate broke some records, discrimination decreased, and participation of women, young individuals, and other minority groups increased in the labor market. At least three factors may be implicated in this movement (Arbache, 2012a). First, the quick demographic transformation Brazil has gone through, which has slowed down the growth rate of the economically active population. Second, the high growth of the service sector which, in general, is highly intensive in terms of unskilled workforce. Third, Brazilian labor laws, which straitjacket the labor market, chiefly in an environment with a lack of skilled labor, as was the case of Brazil in the period. Labor market changes were not homogeneous across sectors. Driven by the increase in family income, regional development, and natural protection barriers, the service sector increased its labor force participation - in 2015, the sector accounted for more than $50 \%$ of new formal work opportunities in the private sector, twice as high as the new job openings in 2002. The service and trade sectors together accounted for over $74 \%$ of new job openings at the end of the decade. Industry and agriculture had a poor labor force participation. Extractive industries increased their participation, but hiring was down in absolute terms.

The economy has been put through profound transformation. The exchange rate had strong appreciation after 2006 and import penetration increased, followed by stagnation or drop in the export rates of several industrial sectors. At the beginning of the decade, industrial products accounted for most of the exports, but in 2011, their share decreased to slightly over one-third, and commodities, on the other hand, was the dominant sector regarding Brazil's participation in the international trade. The share of the industrial sector in value added 
declined, whereas the service and commodities sectors gained momentum in the economy.

In the international scenario, the decade was characterized by strong increases in commodity prices, the outbreak of the international financial crisis in 2008/2010 and its massive deleterious impacts on investments, capital flow, trade, and production. In addition, there were price and market volatilities and, as a response to the crisis, generalized intervention of countries in the economy and the adoption of highly unorthodox fiscal and monetary policies.

Countercyclical policies introduced in Brazil in response to the crisis affected the sectors of activity in different ways. The upswing in civil construction is the most emblematic example.

Given the nature and simultaneity of economic changes in the past decade and their potential impacts on the labor market, it seems reasonable to assume that, despite the stylized fact that wage structure is quite stable (Krueger \& Summers, 1987, 1988; Allen, 1995; Arbache, Dickerson, \& Green, 2004a), there have been non-negligible changes in relative wages.

This analysis is important because it contributes, on the one hand, to the understanding of labor market dynamics and segmentation, wage inequality, and price setting. On the other hand, by identifying the sectors on the winning and losing ends based on the wage premium index, this analysis sheds further light on perspectives of competition at the sectoral level and it is useful for the formulation of industrial, technological, human capital, investment, and foreign trade policies. This paper is structured as follows. Section 2 looks at interindustry wage premiums. Section 3 describes the method, data, and descriptive statistics. Section 4 presents and assesses interindustry wage premiums at the one-digit and two-digit levels. Section 5 investigates whether the labor market would have been more or less segmented. Section 6 explores the behavior and characteristics of the wage structure. Section 7 looks at the relationship between wage premium and competition. Section 8 concludes.

\section{Literature Review}

Research into interindustry wage differentials has focused on attempts to contrast competition theories with efficiency wage theories (Krueger \& Summers, 1988; Katz \& Summers, 1989; Murphy \& Topel, 1990; Arbache, 2001). The most common empirical results are: (i) the individual's and firm's control variables are not enough to explain the wage differential; (ii) interindustry wage differentials are persistent over time; (iii) there is strong correlation between wage premium per occupation and firm size and characteristics in the same industry; (iv) efficiency wage models explain, at least in part, the wage differential; (v) certain industries pay all workers high wages, whereas other industries pay all workers (from CEOs to unskilled workers) low wages. The basic conclusion of the literature is that industrial affiliation is the main source of wage dispersion.

Recently, Jayanthakumaran, Sangkaew and O'Brien (2013) investigate trade-related industrial wage premiums from Thailand. They used the methodology of Krueger and Summers (1988), a two-stage estimation procedure to estimate industry wage premiums after controlling for workers' characteristics and linking wage premiums with explanatory variables that reflect the labor market and trade liberalization. After controlling for observable worker characteristics, wage premiums were found to be high in industries identified as capital and technology intensive such as petroleum and chemical products, presumably requiring more skilled workers. The presence of a positive effect of firm size on workers' wages is well documented in the economic literature (Oi \& Idson, 1999a). Edin and Zetterberg (1992) and Arai (1994) find considerable differences in wage levels between industries when controlling for the characteristics of individuals and firms. According to Idson and Oi (1992) the theory and evidence is persuasive and supports the conclusion that companies that reach large sizes create jobs (technologies, equipment and work organizations) that must be matched with more productive individuals.

For Brazil, the literature has also described great impact of industrial affiliation on wage differential (Arbache, 2001; Arbache, Dickerson, \& Green 2004b, 2001; Arbache \& De Negri, 2004; Arbache \& Corseuil, 2004; inter alia).

Increasing returns to scale, innovation intensity, technology absorption, changes in market competition structure (e.g., competition with China), and government incentives are among the possible causes for the changes in the structure of interindustry wage premiums in open economies, such as Brazil. Whichever the cause, ceteris paribus, the change in relative wages will lead to reallocation of resources at the sectoral level.

If interindustry wage premiums result from factors such as technology, capital stock per worker, increasing returns, and profitability, they can be viewed as indicators of competitive potential. Accordingly, theories such as the strategic trade policy (Eaton \& Grossman, 1986; Brander, 1995; Spencer \& Brander, 2008) provide justification for public interventions that promote the development of industries with competitive potential in oligopolies. 
More recently, partly as a response to the financial crisis and to China's state capitalism policies, justifications have been provided for vertical public interventions combined with horizontal interventions in favor of growth-enhancing sectors. This category includes sectors with high potential for increased productivity, competition, exports, technological innovation, and welfare (e.g., Nunn \& Trefler, 2010; Aghion et al., 2011). If wage premiums capture, at least in part, this potential, then policies should benefit sectors that demonstrate potential increase in wage premium and job creation.

The most usual objective of the wage differential literature is to test the hypothesis of the law of one price and its effect on wage determination. In a competitive labor market, workers with identical characteristics should be paid the same wages and industrial affiliation should not affect wages. In this case, interindustry wage dispersion should be very small or close to zero, since, in principle, one should not expect an employer to pay workers with similar production more than the market wage. Occasional wage differences would be related to differences in workers' production characteristics, working conditions, occupation, etc., but not to industrial affiliation.

Alternative wage determination theories abound, seeking to explain the persistent wage differential.

A good wage determination theory, however, should be able to explain why an employer pays a certain employee more, as this employee could be replaced with another one with a lower wage. The answer to this question is one of the major goals of efficiency wage models. The rationale is that not only do the characteristics of workers, as proposed by the competitive labor market model, but also labor demand affect wage determination. Moreover, institutional issues, such as trade union action, may have decisive effects on the wage determination process.

It is not possible to classify wage differential theories by order of importance, as one theory could be more appropriate than another one to explain specific labor market phenomena. Furthermore, since labor market phenomena are prone to changes, given that social and economic organization changes over time and is influenced by ever-changing institutions and culture - a theory could be relevant to explain a phenomenon in a given period but irrelevant to explain it in another period and/or context.

In recent decades, the theory of labor market segmentation has been extensively investigated, as an attempt to explain problems such as wage dispersion and structure. Numerous factors have been indicated as sources of segmentation, such as geographic regions, workers' demographic characteristics like sex and skin color, unions, labor market regulation, among others. Nevertheless, even before the boom of market segmentation literature in the 1970s, industrial affiliation had been shown to play a crucial role in wage dispersion.

As a matter of fact, a vast number of studies conducted for countries in different stages of development and types of institutions governing the labor market have pointed out industrial affiliation as the source of wage dispersion (e.g. Dickens \& Katz, 1987; Krueger \& Summers, 1987). This literature underscores that industrial characteristics such as market concentration, capital-labor ratio, profitability, and trade union density have a strong impact on wage determination, changing the perspective of analysis from the supply side to the demand side. The more concentrated the industry, or the more market power held by firms in this industry, the higher the average wages. The most common explanation to the relationship between market concentration, profits, and wage differentials lies in the cost of strikes and other types of protests that affect the normal pace of production operations of firms in concentrated markets, which are usually highly profitable due to monopoly rents. Workers affiliated with industries that adopt expensive technologies and complex production processes are presumably paid higher wages. The rationale behind it is that there is a high degree of complementarity between capital and labor in these sectors and that employee turnover is costly and affects the speed of production.

The rate of union workers in an industry would also affect wage determination. There would be a positive relationship between trade union density and union power to negotiate wages.

Doeringer and Piore (1971) argued that a firm's technology is associated with the domestic labor market. Technologically advanced firms require training that is specific to their needs, which, to a certain extent, are acquired after some time working at the firm. Wages are used to retain workers and to reduce turnover, thus leading to wage differentials. With the advent of efficiency wage models in the 1980s, new emphasis was placed on industrial affiliation as source of wage differential. These models propose an interplay between characteristics of the firm and industry and wage determination, incorporating several aspects of the afore-mentioned theories. The models show that wage differential may stem from an optimal equilibrium.

\section{Method, Data, and Descriptive Statistics}

\subsection{Method}

To assess the behavior of interindustry wage premiums and wage structure, wage determination models based on the Mincerian approach were estimated. The model proposed by Haisken-DeNew and Schmidt (1997) was used. 
This model corrects and improves the method originally developed and popularized by Krueger and Summers (1988). As approached in Haisken-DeNew and Schmidt (1997), this improved procedure provides more meaningful economic interpretation of coefficients that measures the deviation from an overall average rather than from a base category. Following the Haisken-DeNew and Schmidt (1997) procedure, we employ the exact formula to compute both adjusted and weighted standard deviations of transformed inter-industry wage premiums and a summary measure of the overall variability of industry wage

$$
\ln \omega_{i j}=\alpha+\beta X_{i}+\varphi Z_{j}+\varepsilon_{i t}
$$

where $\ln w_{\mathrm{ij}}$ is the natural logarithm of the real hourly wage paid to worker $\boldsymbol{i}$ at industry $\boldsymbol{j} ; \mathrm{X}_{\mathrm{i}}$ is the vector of individual, geographic, and corporate characteristics; $Z_{\mathrm{i}}$ is the vector of dummies for the industry, including all industries; $\boldsymbol{\alpha}$ is the intercept; $\varepsilon_{i f}$ is the stochastic term for unobservable characteristics inherent to wage statistics; $\boldsymbol{\beta}$ and $\boldsymbol{\varphi}$ are the parameter values. As all industry dummies are included in the model, a linear restriction $\boldsymbol{j}$ is imposed as follows:

$$
\sum_{j} \omega_{j} \eta_{j}
$$

where $\eta_{j}$ stands for workers' participation at industry $\mathrm{j}$. The estimated coefficients indicate the proportional wage difference for a worker at industry $\mathrm{j}$ and for the average worker representative of the whole set of industries analyzed. The coefficients are directly interpretable and have accurate standard errors.

The standard deviation of the wage differential is calculated as follows:

$$
S D(\varphi)=\sqrt{\eta^{\prime}\left(\bar{H}\left(\varphi_{j}\right)\right) \varphi-\eta^{\prime} \bar{D}\left(V\left(\varphi_{j}\right)\right)}
$$

where $\sigma_{j}^{2}$ is the variance of the estimator, $\varphi$. $S D$ provides the adjusted and weighted standard deviation of the coefficients of industries, $H($.) transforms the column vector into a diagonal matrix, whose diagonal is given by the column vector; $D$ denotes the column vector formed by the diagonal matrix elements; and $V$ is the variance-covariance matrix.

This technique allows checking whether industrial affiliation is relevant in explaining wages beyond individual, geographic, and corporate characteristics. In other words, it allows identifying a "premium" for being associated with a sector. This premium may be positive or negative, and variables such as technology, market structure, value added, productivity associated with skills that were not measured or captured by conventional statistics, but perceived by firms during recruitment and/or retention of human resources, would be the sources of these premiums.

\subsection{Data}

National Household Survey (PNAD) data from 2002 to 2015 were used. To minimize measurement bias and sampling errors associated with unobserved variables, the data were filtered as follows: men, aged 18 to 65 years, in the formal sector, with wage from the major job (full-time). The wages were deflated by the National Consumer Price Index (INPC). The variables are described in Table A1 in the Appendix. The sample comprised 689,206 individuals and the average sample per year included 52,860 workers. Industrial affiliation disaggregated data at the one-digit and two-digit levels were used. The paper focuses on two-digit data.

\subsection{Descriptive Statistics}

Table 1 shows the descriptive statistics for the main variables. Table 2 shows the variables included in the article and their identifications.

Table 1. Estatísticas descritivas

\begin{tabular}{llllll}
\hline Variáveis & Média & DP & Variáveis & Média & DP \\
\hline Salário & 1116,46 & 1388,79 & Norte & 0,09 & 0,29 \\
Grau1 & 0,3 & 0,46 & Nordeste & 0,21 & 0,41 \\
Grau2 & 0,5 & 0,5 & Sudeste & 0,38 & 0,48 \\
Grau3 & 0,14 & 0,35 & Sul & 0,21 & 0,41 \\
Posgrad & 0,01 & 0,08 & Coeste & 0,11 & 0,31 \\
Exper & 18,44 & 12,01 & Tenuy & 4,21 & 5,76 \\
Exper2 & 484,24 & 563,61 & Metrop & 0,49 & 0,50 \\
Sind & 0,24 & 0,43 & Urban & 0,96 & 0,19 \\
Branca & 0,51 & 0,5 & & & \\
\hline
\end{tabular}


Table 2. Variables included in the article

\begin{tabular}{llll|}
\hline Agricultural & AGR & Extraction of non-metallic minerals & ENM \\
\hline Food-Drinks & FDK & Electricity, gas and hot water & EGW \\
\hline Smoke & SMK & Collection, treatment and distribution. Water & CTDW \\
\hline Textiles & TXT & Construction & COM \\
\hline Clothing & CLT & Vehicle trade and repair & VTR \\
\hline Leathers & LEA & Trade intermediaries & TIN \\
\hline Wood & WOO & Accommodation and food & ACF \\
\hline Cellulose-Paper & CEP & Transport, Warehousing and Communication & TWC \\
\hline Edition-Print & EDP & Water transportation & WTR \\
\hline Oil-Alcohol & OAL & Air Transport & ATR \\
\hline Chemicals & CHE & Activ. aux. of transp. ag. on a trip & ATT \\
\hline Rubber-Plastic & RPL & Post and telecommunications & PCM \\
\hline Non-Metallic Minerals & NMM Education & EDU \\
\hline Basic Metallurgy & BME & Health and social services & HSS \\
\hline Metal Products & MPR & Urban cleaning and sewage; & UCS \\
\hline Machines-Equipment & MEQ & Associative activities & AAC \\
\hline Equip. IT & EIT & Recreational, cultural activities & RCA \\
\hline Machines, Apar. Electrical & MAE & Personal services & PSE \\
\hline Apar.Equip. Communications & AEC & Interm. Financ.seg. and previd. Priv. & IFP \\
\hline Equip. from instrum.hospitalares & EIH & Insurance and private pension & IPF \\
\hline Auto-vehicles & AVE & Aux. Activities interm. financial & ATF \\
\hline Equip. Transport & ETR & Real estate activities & RME \\
\hline Miscellaneous Furniture and Industries & MFI & Car rental, machine. and equip. & CME \\
\hline Recycling & REC & Computer activities & CAC \\
\hline Extraction of mineral coal & EMC & Research and Development & RDE \\
\hline Oil extraction & OEX & Services provided to companies & SPC \\
\hline
\end{tabular}

Source: PNAD/IBGE.

Table 3. Participation in employment (\%) - 1 digit

\begin{tabular}{|c|c|c|c|c|c|c|c|c|c|c|c|c|c|}
\hline Sectors & 2002 & 2003 & 2004 & 2005 & 2006 & 2007 & 2008 & 2009 & 2011 & 2012 & 2013 & 2014 & 2015 \\
\hline Agriculture & 1,7 & 1,8 & 1,9 & 1,8 & 1,7 & 1,6 & 1,7 & 1,5 & 2,4 & 2,2 & 2 & 2 & 1,9 \\
\hline Extractive industry & 0,6 & 0,7 & 0,8 & 0,7 & 0,8 & 0,7 & 0,7 & 0,8 & 0,3 & 0,3 & 0,3 & 0,3 & 0,3 \\
\hline Transfor.n industry & 15,9 & 15,7 & 16,4 & 15,8 & 15,5 & 15,7 & 15,3 & 14,3 & 15,9 & 16,1 & 15,9 & 15,7 & 15,4 \\
\hline Construction & 6,3 & 5,4 & 5,7 & 5,7 & 6 & 6,2 & 7,4 & 7,4 & 5,8 & 5,9 & 6,2 & 6,2 & 6 \\
\hline Business & 19,2 & 20 & 18,9 & 19,9 & 20 & 20,3 & 19,4 & 20,2 & 15 & 14,8 & 14,7 & 15 & 14,9 \\
\hline Accommod. and food & 3,4 & 3,4 & 3,4 & 3,4 & 3,5 & 3,7 & 3,4 & 3,6 & 4,9 & 4,7 & 4,6 & 4,7 & 4,9 \\
\hline Tranp, store. Communic & 12,9 & 12,6 & 12,7 & 12,1 & 12,2 & 12,1 & 12,4 & 12,2 & 14,6 & 15 & 16 & 15,9 & 16,5 \\
\hline Education and health & 5,4 & 5,6 & 5,4 & 5,4 & 5,4 & 5,2 & 5,4 & 5,5 & 6,6 & 6,6 & 6,6 & 6,5 & 6,5 \\
\hline Financial intermed. & 29,1 & 29,1 & 29 & 29,7 & 29,4 & 28,9 & 28,7 & 29,5 & 27,7 & 27,8 & 27,1 & 27,4 & 27,3 \\
\hline Subtotal & 94,5 & 94,3 & 94,2 & 94,5 & 94,5 & 94,4 & 94,4 & 95 & 93,2 & 93,4 & 93,4 & 93,7 & 93,7 \\
\hline Industries not includ. & 5,5 & 5,7 & 5,8 & 5,5 & 5,5 & 5,6 & 5,6 & 5 & 6,8 & 6,6 & 6,6 & 6,3 & 6,3 \\
\hline
\end{tabular}

Source: PNAD/IBGE.

Tables 3 and 4 show the labor structure at the one-digit and two-digit levels, respectively. In general, the labor force participation of sectors remained relatively stable, but important changes occurred. The participation of the industrial and agricultural sectors waned, whereas that of the mineral extraction, civil construction, and service sectors increased. Table 5 and 6 show the behavior of real wages at the two-digit and one-digit levels. It is clear that real wages also rose substantially at this level, but wage dispersion decreased. In fact, the coefficient of variation dropped from 1.28 in 2002 to 1.10 in 2015, suggesting a tendency towards lower wage dispersion over time. Figure 1 shows the average productivity of Brazilian workers during the study period. Labor productivity also varied considerably across sectors. Productivity in the processing industry grew on average $0.9 \%$ per year between 2002 and 2015. Productivity rose $0.5 \%$ per year in the service sector; $1.8 \%$ in the extractive sector; and $4.3 \%$ in the agricultural sector (Ipea, 2012). The Brazilian economic period was characterized in the 2000s by significant structural changes in relation to previous decades, many of which were driven by the foreign market, the labor market and, mainly, the economic stabilization policy.

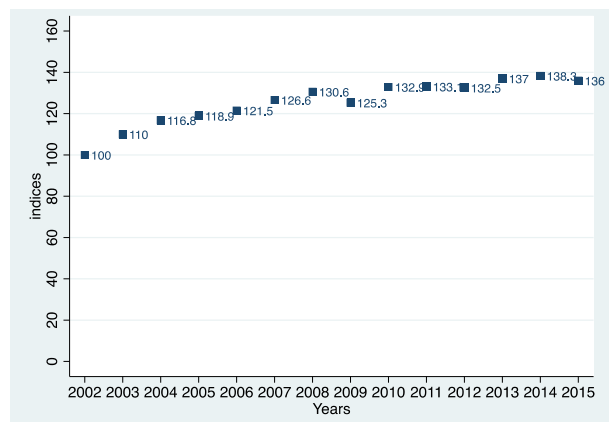

Figure 1. Worker productivity index in industry Brazil: 2002-2015 
Table 4. Employment participation (\%) - 2 digits

\begin{tabular}{|c|c|c|c|c|c|c|c|c|c|c|c|c|c|}
\hline Industrial & 2002 & 2003 & 2004 & 2005 & 2006 & 2007 & 2008 & 2009 & 2011 & 2012 & 2013 & 2014 & 2015 \\
\hline Agricultural & 8,84 & 9,24 & 9,47 & 9,39 & 8,77 & 8,23 & 8,5 & 7,86 & 8,74 & 8,07 & 8,05 & 8,08 & 7,54 \\
\hline Food-Drinks & 4,29 & 4,05 & 4,14 & 4,11 & 4,44 & 4,34 & 4,35 & 4,27 & 1,32 & 1,42 & 1,4 & 1,3 & 1,28 \\
\hline Smoke & 0,06 & 0,07 & 0,07 & 0,04 & 0,03 & 0,05 & 0,05 & 0,04 & 0,02 & 0,02 & 0,02 & 0,02 & 0,01 \\
\hline Textiles & 1,37 & 0,98 & 1,05 & 1,06 & 0,99 & 0,94 & 0,98 & 0,92 & 0,45 & 0,49 & 0,51 & 0,52 & 0,46 \\
\hline Clothing & 0,59 & 0,7 & 0,72 & 0,78 & 0,70 & 0,78 & 0,69 & 0,70 & 1,15 & 1,36 & 1,25 & 1,15 & 1,04 \\
\hline Leathers & 2,03 & 2,15 & 2,04 & 2,00 & 1,90 & 1,88 & 1,56 & 1,63 & 0,52 & 0,49 & 0,46 & 0,44 & 0,39 \\
\hline wood & 1,93 & 1,76 & 1,79 & 1,68 & 1,52 & 1,32 & 1,05 & 1,09 & 0,33 & 0,26 & 0,28 & 0,4 & 0,35 \\
\hline Cellulose-Paper & 0,64 & 0,68 & 0,68 & 0,61 & 0,65 & 0,7 & 0,64 & 0,50 & 0,17 & 0,17 & 0,17 & 0,16 & 0,15 \\
\hline Edition-Print & 0,98 & 0,9 & 0,87 & 0,89 & 0,87 & 0,86 & 0,86 & 0,84 & 0,23 & 0,23 & 0,22 & 0,2 & 0,21 \\
\hline Oil-Alcohol & 0,44 & 0,45 & 0,54 & 0,41 & 0,41 & 0,65 & 0,64 & 0,72 & 0,15 & 0,13 & 0,11 & 0,14 & 0,1 \\
\hline Chemicals & 1,95 & 2,36 & 2,41 & 2,02 & 2,16 & 2,38 & 1,61 & 1,58 & 0,4 & 0,36 & 0,4 & 0,42 & 0,39 \\
\hline Rubber-Plastic & 1,09 & 1,1 & 1,1 & 1,2 & 1,13 & 1,22 & 1,08 & 1,03 & 0,2 & 0,23 & 0,2 & 0,23 & 0,24 \\
\hline Non-Metallic Minerals & 0,16 & 0,18 & 0,18 & 0,2 & 0,16 & 0,17 & 0,2 & 0,13 & 0,04 & 0,05 & 0,05 & 0,05 & 0,04 \\
\hline Basic Metallurgy & 1,26 & 1,39 & 1,31 & 1,4 & 1,49 & 1,39 & 2,08 & 1,58 & 0,31 & 0,25 & 0,25 & 0,25 & 0,2 \\
\hline Metal Products & 2,11 & 2,1 & 2,12 & 2,05 & 2,08 & 2,41 & 2,12 & 1,72 & 0,51 & 0,49 & 0,48 & 0,49 & 0,48 \\
\hline Machines-Equipment & 2,01 & 2,38 & 2,54 & 2,42 & 2,19 & 2,37 & 1,95 & 2,39 & 0,56 & 0,57 & 0,58 & 0,54 & 0,49 \\
\hline Equip. IT & 0,09 & 0,11 & 0,11 & 0,1 & 0,09 & 0,11 & 0,11 & 0,11 & 0,03 & 0,02 & 0,03 & 0,03 & 0,02 \\
\hline Machines, Apar. Electrical & 0,39 & 0,41 & 0,53 & 0,54 & 0,49 & 0,39 & 0,62 & 0,52 & 0,11 & 0,1 & 0,09 & 0,11 & 0,09 \\
\hline Apar.Equip. Communications & 0,34 & 0,34 & 0,47 & 0,41 & 0,45 & 0,46 & 0,28 & 0,25 & 0,06 & 0,06 & 0,06 & 0,06 & 0,06 \\
\hline Equip. from instrum.hospitalares & 0,25 & 0,27 & 0,28 & 0,24 & 0,28 & 0,22 & 0,23 & 0,20 & 0,08 & 0,07 & 0,06 & 0,07 & 0,06 \\
\hline Auto-vehicles & 1,7 & 1,67 & 1,73 & 1,84 & 1,76 & 1,77 & 1,95 & 1,73 & 0,41 & 0,5 & 0,47 & 0,4 & 0,33 \\
\hline Equip. Transport & 0,39 & 0,4 & 0,46 & 0,43 & 0,49 & 0,41 & 0,45 & 0,52 & 0,09 & 0,11 & 0,11 & 0,12 & 0,12 \\
\hline Miscellaneous Furniture and Industries & 1,7 & 1,39 & 1,43 & 1,48 & 1,31 & 1,33 & 1,31 & 1,15 & 0,71 & 0,69 & 0,6 & 0,47 & 0,46 \\
\hline Recycling & 0,05 & 0,1 & 0,14 & 0,14 & 0,12 & 0,12 & 0,19 & 0,15 & 0,05 & 0,06 & 0,06 & 0,07 & 0,06 \\
\hline Extraction of mineral coal & 0,01 & 0,01 & 0,01 & 0,02 & 0,00 & 0,01 & 0,01 & 0,01 & 0,01 & 0,01 & 0,01 & 0,01 & 0,01 \\
\hline Oil extraction & 0,13 & 0,2 & 0,28 & 0,19 & 0,19 & 0,18 & 0,21 & 0,33 & 0,07 & 0,09 & 0,06 & 0,09 & 0,07 \\
\hline Extraction of metallic minerals & 0,25 & 0,21 & 0,36 & 0,25 & 0,40 & 0,3 & 0,44 & 0,41 & 0,09 & 0,12 & 0,12 & 0,12 & 0,1 \\
\hline Extraction of non-metallic minerals & 0,42 & 0,52 & 0,48 & 0,55 & 0,49 & 0,67 & 0,47 & 0,53 & 0,14 & 0,1 & 0,11 & 0,09 & 0,08 \\
\hline Electricity, gas and hot water & 0,6 & 0,64 & 0,68 & 0,63 & 0,77 & 0,59 & 0,58 & 0,63 & 0,17 & 0,17 & 0,17 & 0,18 & 0,17 \\
\hline Collection, treatment and distribution. Water & 0,11 & 0,2 & 0,24 & 0,12 & 0,15 & 0,17 & 0,15 & 0,19 & 0,12 & 0,1 & 0,11 & 0,13 & 0,12 \\
\hline Construction & 8,43 & 7,21 & 7,57 & 7,65 & 8,08 & 8,35 & 9,91 & 10,01 & 6,15 & 6,35 & 6,63 & 6,76 & 6,25 \\
\hline Vehicle trade and repair & 5,3 & 5,28 & 4,99 & 5,25 & 5,33 & 5,46 & 5,47 & 5,42 & 2,33 & 2,36 & 2,35 & 2,41 & 2,34 \\
\hline Trade intermediaries & 15,23 & 16,16 & 15,06 & 16,04 & 16,12 & 16,24 & 15,3 & 16,51 & 10,35 & 10,36 & 10,14 & 10,58 & 10,13 \\
\hline Accommodation and food & 3,02 & 3,05 & 3,01 & 3,06 & 3,12 & 3,26 & 3,04 & 3,22 & 4,94 & 4,79 & 4,65 & 4,8 & 5,02 \\
\hline Transport, Warehousing and Communication & 7,34 & 7,17 & 7,09 & 6,77 & 6,95 & 6,68 & 6,93 & 6,74 & 3,06 & 3,11 & 3,11 & 3,1 & 2,98 \\
\hline Water transportation & 0,2 & 0,18 & 0,16 & 0,16 & 0,16 & 0,15 & 0,17 & 0,20 & 0,06 & 0,06 & 0,06 & 0,07 & 0,05 \\
\hline Air Transport & 0,26 & 0,23 & 0,24 & 0,22 & 0,16 & 0,19 & 0,15 & 0,22 & 0,07 & 0,07 & 0,07 & 0,06 & 0,05 \\
\hline Activ. aux. of transp. ag. on a trip & 0,86 & 0,92 & 0,84 & 0,77 & 0,86 & 1,02 & 0,94 & 0,99 & 0,32 & 0,33 & 0,33 & 0,35 & 0,38 \\
\hline Post and telecommunications & 1,19 & 1,17 & 1,27 & 1,31 & 1,17 & 1,18 & 1,26 & 1,30 & 0,46 & 0,48 & 0,42 & 0,44 & 0,4 \\
\hline Education & 1,82 & 1,8 & 1,78 & 1,87 & 1,71 & 1,76 & 1,81 & 1,86 & 4,05 & 4,14 & 4,38 & 4,42 & 4,34 \\
\hline Health and social services & 1,36 & 1,55 & 1,42 & 1,42 & 1,49 & 1,34 & 1,39 & 1,42 & 2,8 & 3,01 & 3,19 & 3,24 & 3,34 \\
\hline Urban cleaning and sewage; & 0,46 & 0,48 & 0,5 & 0,48 & 0,42 & 0,44 & 0,45 & 0,48 & 0,1 & 0,09 & 0,14 & 0,15 & 0,13 \\
\hline Associative activities & 0,4 & 0,46 & 0,48 & 0,39 & 0,44 & 0,44 & 0,42 & 0,46 & 0,33 & 0,39 & 0,31 & 0,37 & 0,32 \\
\hline Recreational, cultural activities & 1,41 & 1,28 & 1,36 & 1,23 & 1,25 & 1,17 & 1,28 & 1,21 & 0,85 & 0,82 & 0,81 & 0,88 & 0,85 \\
\hline Personal services & 0,25 & 0,31 & 0,28 & 0,3 & 0,36 & 0,3 & 0,32 & 0,38 & 1,44 & 1,54 & 1,55 & 1,68 & 1,63 \\
\hline Interm. Financ.seg. & 1,41 & 1,43 & 1,22 & 1,07 & 1,18 & 1,21 & 1,12 & 1,01 & 0,67 & 0,64 & 0,68 & 0,68 & 0,63 \\
\hline Insurance and private pension & 0,29 & 0,33 & 0,25 & 0,24 & 0,21 & 0,28 & 0,33 & 0,25 & 0,09 & 0,09 & 0,09 & 0,1 & 0,09 \\
\hline Aux. Activities interm. financial & 0,15 & 0,2 & 0,23 & 0,18 & 0,18 & 0,19 & 0,14 & 0,18 & 0,15 & 0,17 & 0,17 & 0,15 & 0,13 \\
\hline Real estate activities & 2,49 & 2,32 & 2,48 & 2,36 & 2,30 & 2,17 & 1,85 & 2,29 & 0,64 & 0,7 & 0,71 & 0,71 & 0,69 \\
\hline Car rental, machine. and equip. & 0,22 & 0,24 & 0,17 & 0,23 & 0,20 & 0,2 & 0,16 & 0,25 & 0,09 & 0,08 & 0,09 & 0,09 & 0,08 \\
\hline Computer activities & 0,63 & 0,68 & 0,66 & 0,75 & 0,78 & 0,84 & 0,86 & 0,83 & 0,43 & 0,43 & 0,39 & 0,4 & 0,4 \\
\hline Research and Development & 0,07 & 0,05 & 0,02 & 0,04 & 0,04 & 0,04 & 0,05 & 0,04 & 0,03 & 0,04 & 0,04 & 0,04 & 0,03 \\
\hline Services provided to companies & 7,72 & 7,73 & 7,75 & 8,37 & 8,20 & 7,93 & 8,25 & 8,49 & 4,14 & 4,23 & 3,89 & 4,1 & 3,95 \\
\hline
\end{tabular}

Source:PNAD/IBGE. 
Table 5. Average real wages ( $R$ \$) 2 digits

\begin{tabular}{|c|c|c|c|c|c|c|c|c|c|c|c|c|c|}
\hline Sectors & 2002 & 2003 & 2004 & 2005 & 2006 & 2007 & 2008 & 2009 & 2011 & 2012 & 2013 & 2014 & 2015 \\
\hline Agricultural & 547 & 617 & 653 & 728 & 744 & 725 & 819 & 885 & 1132 & 1250 & 1380 & 1367 & 1528 \\
\hline Food-Drinks & 605 & 644 & 633 & 703 & 815 & 844 & 900 & 944 & 1130 & 1291 & 1384 & 1530 & 1561 \\
\hline Smoke & 540 & 622 & 967 & 784 & 1458 & 2105 & 1693 & 1620 & 1511 & 1562 & 2216 & 1475 & 2031 \\
\hline Textiles & 561 & 625 & 709 & 718 & 820 & 861 & 891 & 922 & 1111 & 1208 & 1371 & 1379 & 1488 \\
\hline Clothing & 447 & 516 & 538 & 629 & 689 & 763 & 771 & 810 & 903 & 1104 & 1193 & 1266 & 1256 \\
\hline Leathers & 496 & 532 & 618 & 683 & 826 & 712 & 789 & 793 & 941 & 1005 & 1167 & 1218 & 1272 \\
\hline wood & 516 & 651 & 618 & 714 & 726 & 851 & 911 & 1041 & 1289 & 1378 & 1576 & 1715 & 1581 \\
\hline Cellulose-Paper & 779 & 1023 & 1016 & 923 & 1014 & 1107 & 1246 & 1531 & 1399 & 1508 & 1776 & 2057 & 2208 \\
\hline Edition-Print & 1131 & 1138 & 1078 & 1188 & 1294 & 1370 & 1387 & 1497 & 1783 & 1951 & 2278 & 2401 & 2212 \\
\hline Oil-Alcohol & 1342 & 1251 & 1168 & 1456 & 1982 & 1682 & 2066 & 1684 & 1953 & 2765 & 2075 & 2960 & 2719 \\
\hline Chemicals & 1116 & 1098 & 1108 & 1622 & 1439 & 1354 & 1516 & 1754 & 1960 & 2289 & 2416 & 2581 & 2786 \\
\hline Rubber-Plastic & 697 & 926 & 887 & 1024 & 1057 & 957 & 1094 & 1196 & 1206 & 1761 & 1737 & 2121 & 1828 \\
\hline Non-Metallic Minerals & 878 & 759 & 931 & 959 & 837 & 1165 & 924 & 1558 & 1353 & 1494 & 1852 & 1387 & 1766 \\
\hline Basic Metallurgy & 905 & 1050 & 1038 & 1438 & 1088 & 1278 & 1385 & 1471 & 1772 & 2003 & 2269 & 2173 & 2240 \\
\hline Metal Products & 806 & 848 & 910 & 996 & 991 & 1115 & 1150 & 1296 & 1393 & 1664 & 1879 & 1869 & 2173 \\
\hline Machines-Equipment & 970 & 1000 & 1039 & 1179 & 1295 & 1377 & 1388 & 1481 & 1654 & $=1756$ & 1863 & 2021 & 2208 \\
\hline Equip. IT & 1309 & 1299 & 1140 & 1431 & 1678 & 1280 & 2267 & 1346 & 1724 & 2021 & 3227 & 2564 & 2974 \\
\hline Machines, Apar. Electrical & 975 & 1216 & 1095 & 1386 & 1045 & 1192 & 1232 & 1294 & 1679 & 1789 & 1836 & 1975 & 2263 \\
\hline Apar.Equip. Communications & 1056 & 860 & 909 & 1052 & 1123 & 1198 & 1211 & 1059 & 1585 & 1674 & 1732 & 1909 & 1757 \\
\hline Equip. from instrum. hospitalares & 954 & 1054 & 1095 & 1175 & 1348 & 1454 & 1652 & 1757 & 1916 & 2152 & 2260 & 2328 & 2983 \\
\hline Auto-vehicles & 1004 & 1144 & 1304 & 1214 & 1418 & 1427 & 1606 & 1717 & 1882 & 1973 & 2201 & 2454 & 2617 \\
\hline Equip. Transport & 947 & 965 & 1076 & 1331 & 1338 & 1371 & 1418 & 1782 & 1663 & 1742 & 1928 & 2418 & 2262 \\
\hline Miscellaneous Fur & 640 & 684 & 708 & 803 & 847 & 955 & 958 & 1064 & 1287 & 1414 & 1587 & 1686 & 1777 \\
\hline Recycling & 526 & 614 & 598 & 714 & 569 & 680 & 742 & 1129 & 1263 & 1184 & 1281 & 1426 & 1271 \\
\hline Extraction of mineral coal & 746 & 1296 & 792 & 1074 & 2319 & 1402 & 1575 & 4600 & 1294 & $=1334$ & 2359 & 1833 & 3962 \\
\hline Oil extraction & 2146 & 2601 & 2249 & 2524 & 3106 & 2828 & 3330 & 3360 & 3729 & 4634 & 5195 & 5086 & 6606 \\
\hline Extraction of metallic minerals & 868 & 824 & 1151 & 1094 & 1289 & 1309 & 1691 & 1719 & 2103 & 2549 & 2899 & 2855 & 2897 \\
\hline Extraction of non-metallic minerals & 775 & 906 & 1151 & 1081 & 1122 & 1268 & 1418 & 1836 & 1492 & 1879 & 2015 & 1906 & 2244 \\
\hline Electricity, gas and hot water & 1309 & 1516 & 1551 & 1740 & 1890 & 1997 & 2238 & 2227 & 2422 & 2486 & 2977 & 3246 & 3299 \\
\hline Collection, treatment and distribution. Water & 1024 & 1187 & 1192 & 1409 & 1448 & 1683 & 1451 & 1499 & 2174 & 2212 & 2289 & 2914 & 2553 \\
\hline Construction & 736 & 766 & 819 & 939 & 1006 & 1043 & 1086 & 1141 & 1367 & 1563 & 1736 & 1803 & 1985 \\
\hline Vehicle trade and repair & 783 & 891 & 905 & 970 & 1051 & 1096 & 1243 & 1298 & 1452 & 1648 & 1699 & 1905 & 1868 \\
\hline Trade intermediaries & 761 & 783 & 841 & 912 & 981 & 1010 & 1098 & 1114 & 1329 & 1462 & 1567 & 1677 & 1715 \\
\hline Accommodation and food & 556 & 612 & 719 & 760 & 800 & 824 & 875 & 940 & 1126 & 1258 & 1352 & 1408 & 1512 \\
\hline Transport, Warehousing and Communication & 794 & 881 & 956 & 982 & 1070 & 1158 & 1278 & 1338 & 1454 & $=1616$ & 1759 & 1847 & 1874 \\
\hline Water transportation & 834 & 994 & 1053 & 1324 & 1418 & 1430 & 1861 & 1702 & 2105 & 2580 & 2426 & 2872 & 3421 \\
\hline Air Transport & 1622 & 1729 & 1710 & 1736 & 1955 & 2150 & 2321 & 2554 & 3008 & 3445 & 3696 & 3141 & 3567 \\
\hline Activ. aux. of transp. ag. on a trip & 915 & 952 & 1105 & 1157 & 1178 & 1232 & 1409 & 1540 & 1748 & 1843 & 1830 & 2092 & 2320 \\
\hline Post and telecommunications & 985 & 1020 & 1152 & 1104 & 1201 & 1331 & 1231 & 1521 & 1619 & 1616 & 1842 & 2204 & 2122 \\
\hline Education & 758 & 828 & 886 & 962 & 1072 & 1134 & 1230 & 1356 & 1657 & 1821 & 1986 & 2105 & 2338 \\
\hline Health and social services & 979 & 1082 & 1168 & 1239 & 1319 & 1385 & 1496 & 1626 & 1866 & 2014 & 2280 & 2410 & 2529 \\
\hline Urban cleaning and sewage; & 456 & 438 & 489 & 571 & 592 & 641 & 722 & 742 & 1010 & 1198 & 1049 & 1303 & 1654 \\
\hline Associative activities & 823 & 769 & 958 & 826 & 1110 & 1015 & 1173 & 1311 & 1435 & 1697 & 1812 & 1700 & 2004 \\
\hline Recreational, cultural activities & 932 & 932 & 1037 & 1164 & 1206 & 1492 & 1563 & 1441 & 1859 & 1979 & 2338 & 2532 & 2558 \\
\hline Personal services & 619 & 733 & 677 & 871 & 874 & 974 & 1099 & 1152 & 1324 & $=1384$ & 1405 & 1588 & 1680 \\
\hline Interm. Financ.seg. and previd. Priv. & 1810 & 1781 & 2003 & 2126 & 2238 & 2233 & 2323 & 2570 & 3056 & 3262 & 3637 & 3931 & 4180 \\
\hline Insurance and private pension & 1141 & 1402 & 1221 & 1472 & 1480 & 1715 & 1928 & 1678 & 2052 & 2218 & 2480 & 2649 & 2892 \\
\hline Aux. Activities interm. financial & 1505 & 1383 & 1680 & 1667 & 1529 & 2305 & 2396 & 1850 & 2670 & 3035 & 2619 & 3475 & 3226 \\
\hline Real estate activities & 657 & 612 & 737 & 741 & 734 & 919 & 958 & 1058 & 1476 & 1484 & 1601 & 1713 & 1912 \\
\hline Car rental, machine. and equip. & 870 & 991 & 1036 & 907 & 1340 & 1420 & 1858 & 1426 & 2466 & 2027 & 2481 & 2545 & 2660 \\
\hline Computer activities & 1604 & 1660 & 1815 & 2061 & 1759 & 2083 & 2260 & 2490 & 2730 & 2997 & 3342 & 3694 & 3514 \\
\hline Research and Develor & 1702 & 1944 & 1820 & 2279 & 2371 & 2167 & 2190 & 2932 & 3603 & 3990 & 4459 & 4063 & 4490 \\
\hline Services provided to companies & 933 & 1059 & 1032 & 1131 & 1271 & 1302 & 1382 & 1477 & 1799 & 1979 & 2207 & 2198 & 2344 \\
\hline
\end{tabular}

Source:PNAD/IBGE. 
Table 6. Average real wages (R\$)- 1 digit

\begin{tabular}{|c|c|c|c|c|c|c|c|c|c|c|c|c|c|}
\hline Sectors & 2002 & 2003 & 2004 & 2005 & 2006 & 2007 & 2008 & 2009 & 2011 & 2012 & 2013 & 2014 & 2015 \\
\hline Agriculture & 543 & 606 & 647 & 724 & 728 & 710 & 802 & 874 & 1087 & 1241 & 1345 & 1317 & 1483 \\
\hline indus_extrativ & 1164 & 1359 & 1414 & 1534 & 1731 & 1756 & 1941 & 2082 & 2329 & 2674 & 2914 & 3194 & 3387 \\
\hline Indus_Transf & 732 & 807 & 833 & 952 & 1006 & 1047 & 1129 & 1201 & 1380 & 1538 & 1683 & 1822 & 1894 \\
\hline Constr_Civil & 736 & 766 & 819 & 939 & 1006 & 1043 & 1086 & 1141 & 1367 & 1563 & 1736 & 1803 & 1985 \\
\hline Business & 765 & 803 & 852 & 923 & 994 & 1026 & 1125 & 1147 & 1352 & 1497 & 1591 & 1719 & 1744 \\
\hline Aloj_Alimen & 556 & 612 & 719 & 760 & 800 & 824 & 875 & 940 & 1126 & 1258 & 1352 & 1408 & 1512 \\
\hline Tranp_Arm_Com & 861 & 935 & 1028 & 1046 & 1127 & 1225 & 1308 & 1430 & 1550 & 1691 & 1830 & 1961 & 2004 \\
\hline Educ_Saude & 841 & 925 & 991 & 1067 & 1167 & 1231 & 1335 & 1460 & 1742 & 1902 & 2109 & 2233 & 2420 \\
\hline Serv_Domes & 283 & 318 & 342 & 381 & 429 & 467 & 509 & 565 & 673 & 767 & 841 & 913 & 972 \\
\hline Ser-Col-Soc=Pes & 759 & 781 & 858 & 941 & 1020 & 1142 & 1246 & 1247 & 1515 & 1636 & 1752 & 1905 & 2018 \\
\hline IntFin_Seg_Priv & 1088 & 1171 & 1208 & 1296 & 1388 & 1473 & 1572 & 1645 & 2014 & 2176 & 2413 & 2498 & 2628 \\
\hline
\end{tabular}

Source: PNAD/IBGE.

\section{Interindustry Wage Premiums}

Figure 1 shows the distribution of average wage premiums. The premiums range from $-22.2 \%$ to $75.4 \%$ and are, therefore, quite disperse, thus confirming the stylized fact that the Brazilian labor market is highly segmented.

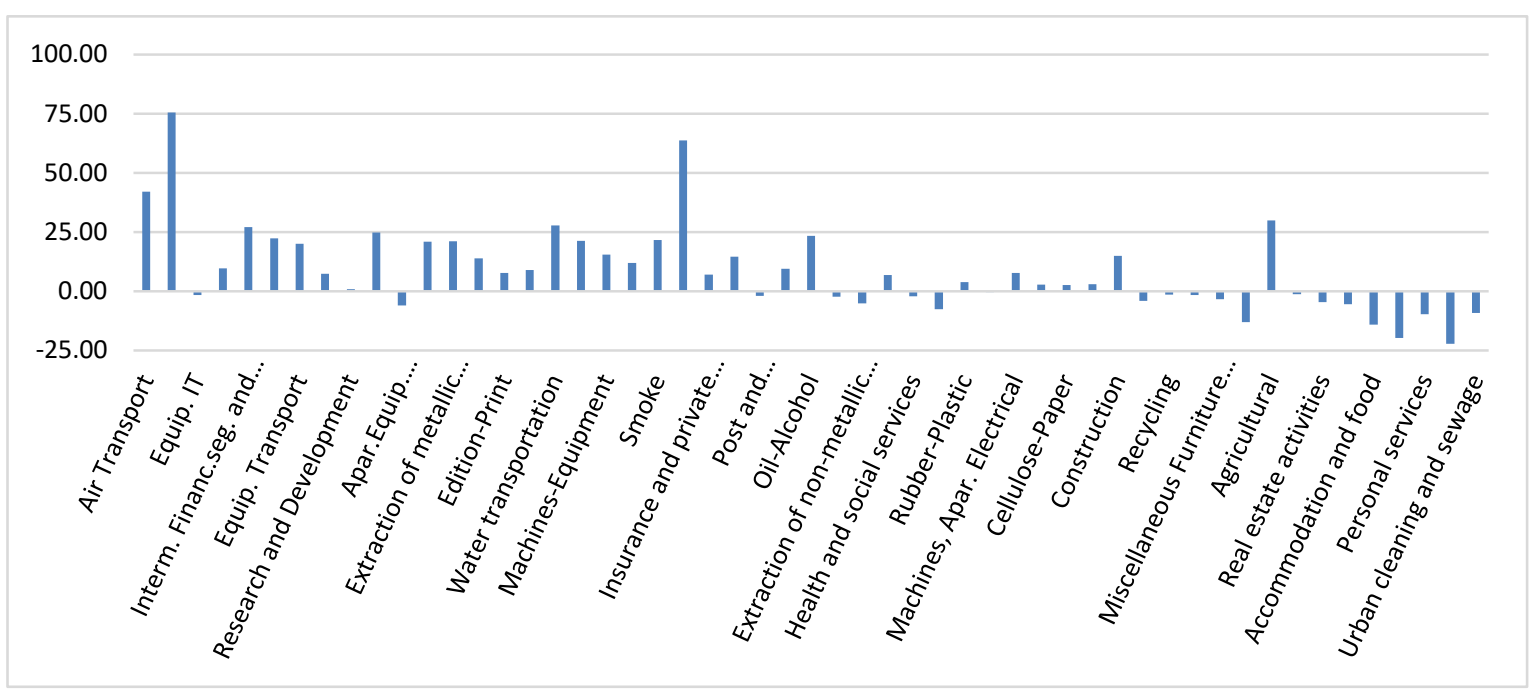

Figure 2. Interindustrial salary premium - 2 digits - with control

Source: PNAD/IBGE.

Table 7 shows controlled wage premiums at the two-digit level. The results underscore that industrial affiliation is the primary source of wage determination and differential. Just like at the one-digit level, the inclusion of controls substantially alters the coefficients, especially the relevance of industrial affiliation. In 2015, a worker in the petroleum extraction sector earned $75.4 \%$ more than other workers with the same characteristics, whereas a worker in the rubber-plastic sector earned $3.79 \%$ less. Note: All coefficients are statistically significant at $1 \%$. The education and health sectors showed an opposite behavior, as wage premiums dropped after the inclusion of controls in the model. The premium plummeted, going from $21.5 \%$, when not controlled for, to less than $-1.31 \%$, when controlled for. 
Table 7. Interindustrial salary premium - 2 digits - with control

\begin{tabular}{|c|c|c|c|c|c|c|c|c|c|c|c|c|c|}
\hline Industries & 2002 & 2003 & 2004 & 2005 & 2006 & 2007 & 2008 & 2009 & 2011 & 2012 & 2013 & 2014 & 2015 \\
\hline Air Transport & 61,13 & 41,08 & 32,97 & 49,52 & 73,31 & 39,66 & 35,18 & 55,01 & 48,80 & 46,80 & 53,10 & 43,30 & 42,00 \\
\hline Oil extraction & 55,60 & 59,46 & 65,49 & 46,72 & 63,24 & 62,22 & 54,88 & 56,20 & 50,20 & 59,00 & 58,80 & 50,40 & 75,40 \\
\hline Equip. IT & 53,93 & 30,58 & 11,84 & 17,11 & 23,00 & 20,93 & 40,94 & 19,73 & 11,70 & 22,70 & 18,30 & 36,80 & $-1,55$ \\
\hline Aux. Activities interm. financial & 50,43 & 10,53 & 22,14 & 25,50 & 9,75 & 12,93 & 32,63 & 20,20 & 9,39 & 12,60 & 14,40 & 11,90 & 9,66 \\
\hline Interm. Financ.seg. and previd. Priv. & 37,66 & 28,40 & 24,70 & 27,42 & 28,17 & 26,22 & 29,82 & 29,64 & 29,10 & 30,70 & 25,10 & 29,50 & 27,00 \\
\hline Computer activities & 35,24 & 27,59 & 32,21 & 28,99 & 17,95 & 21,24 & 25,04 & 27,90 & 32,10 & 29,60 & 27,00 & 30,00 & 22,40 \\
\hline Equip. Transport & 34,88 & 15,52 & 16,23 & 26,48 & 24,43 & 19,06 & 24,89 & 24,07 & 24,80 & 22,20 & 32,20 & 37,70 & 20,10 \\
\hline Equip. from instrum.hospitalares & 34,52 & 13,57 & 18,97 & 15,88 & 20,00 & 10,16 & 15,76 & 24,84 & 14,00 & 7,01 & 11,10 & 10,00 & 7,27 \\
\hline Research and Development & 31,71 & 11,43 & 92,36 & $-4,09$ & $-5,22$ & 10,46 & $-6,97$ & 1,73 & 43,10 & 44,20 & 9,23 & 12,20 & 0,93 \\
\hline Electricity, gas and hot water & 30,44 & 22,32 & 15,21 & 16,43 & 20,60 & 19,49 & 16,80 & 27,88 & 27,80 & 19,00 & 29,80 & 26,90 & 24,80 \\
\hline Apar.Equip. Communications & 28,77 & 16,38 & 16,44 & 19,16 & 16,16 & 16,61 & 12,38 & 10,84 & 7,96 & 19,90 & 19,50 & 25,00 & $-5,96$ \\
\hline Auto-vehicles & 28,13 & 25,16 & 27,41 & 24,66 & 25,34 & 25,99 & 24,04 & 22,63 & 27,60 & 29,30 & 22,90 & 24,60 & 20,90 \\
\hline Extraction of metallic minerals & 27,90 & 22,30 & 31,35 & 20,28 & 34,29 & 23,68 & 38,59 & 36,20 & 46,30 & 46,30 & 44,60 & 44,50 & 21,00 \\
\hline Chemicals & 27,12 & 13,24 & 13,74 & 19,08 & 17,10 & 12,09 & 15,33 & 13,59 & 18,40 & 14,80 & 15,40 & 12,10 & 13,80 \\
\hline Edition-Print & 26,58 & 19,63 & 6,29 & 14,27 & 7,80 & 9,08 & 10,53 & 9,52 & 10,30 & 6,43 & 4,46 & 17,10 & 7,67 \\
\hline Car rental, machine. and equip. & 25,74 & 15,43 & $-4,55$ & 8,30 & 5,24 & 11,16 & 11,90 & 1,05 & 7,02 & 5,08 & 17,30 & $-3,92$ & 8,88 \\
\hline Water transportation & 22,25 & 11,29 & 20,93 & 19,62 & 29,20 & 20,71 & 10,80 & 30,26 & 48,80 & 46,80 & 37,80 & 35,20 & 27,70 \\
\hline Basic Metallurgy & 20,69 & 13,63 & 15,28 & 15,42 & 15,51 & 17,80 & 17,35 & 19,33 & 23,30 & 24,40 & 23,90 & 19,60 & 21,20 \\
\hline Machines-Equipment & 20,57 & 18,20 & 14,61 & 21,22 & 20,15 & 20,26 & 19,30 & 17,06 & 13,80 & 15,60 & 19,40 & 16,90 & 15,40 \\
\hline Metal Products & 20,50 & 8,24 & 12,04 & 9,01 & 10,45 & 11,41 & 12,95 & 12,08 & 14,80 & 14,20 & 12,20 & 15,00 & 11,90 \\
\hline Smoke & 19,79 & 6,36 & 21,72 & 6,48 & 36,36 & 28,91 & 29,31 & 25,01 & 30,80 & 5,40 & 22,20 & $-5,36$ & 21,70 \\
\hline Extraction of mineral coal & 19,76 & 17,63 & 58,15 & 44,86 & 159,13 & $-40,34$ & 48,59 & 19,78 & 23,10 & 43,10 & 25,60 & 29,50 & 63,70 \\
\hline Insurance and private pension & 18,62 & 26,83 & 1,54 & 22,04 & 15,17 & 9,77 & 22,67 & 16,87 & 29,90 & 9,68 & 11,70 & 8,91 & 6,96 \\
\hline Non-Metallic Minerals & 16,93 & 9,19 & 2,20 & $-1,04$ & 3,29 & $-3,26$ & 7,26 & 13,53 & 17,00 & 18,30 & 16,90 & 23,00 & 14,60 \\
\hline Post and telecommunications & 16,82 & 16,13 & 14,19 & 11,51 & 9,89 & 8,34 & 7,20 & 8,71 & 9,19 & 2,45 & 7,19 & 4,23 & $-1,98$ \\
\hline Activ. aux. of transp. ag. on a & 15,93 & $-0,51$ & 8,97 & 3,94 & 4,77 & 7,43 & 3,58 & 5,66 & 9,38 & 3,65 & 5,47 & 2,81 & 9,49 \\
\hline Oil-Alcohol & 15,30 & 10,43 & 11,98 & 21,16 & 15,61 & 23,45 & 24,79 & 25,42 & 26,00 & 30,80 & 19,20 & 35,70 & 23,40 \\
\hline Recreational, cultural activities & 14,93 & 1,95 & 5,16 & 4,70 & 7,52 & 4,14 & 6,92 & 4,83 & 6,67 & 8,37 & 3,91 & 8,93 & $-2,25$ \\
\hline Extraction of non-metallic minerals & 12,87 & 3,94 & 6,38 & 23,64 & 9,79 & 15,00 & 10,42 & 21,64 & 10,10 & 13,10 & 4,05 & 7,28 & $-5,13$ \\
\hline Transport, Warehousing and Commur & 12,47 & 7,23 & 5,61 & 6,38 & 5,66 & 3,83 & 4,29 & 5,28 & 9,97 & 11,20 & 9,60 & 11,00 & 6,80 \\
\hline Health and social services & 12,36 & 5,72 & 9,91 & 4,83 & 6,05 & 2,71 & 4,23 & 9,89 & $-2,25$ & $-1,00$ & $-3,52$ & 1,58 & $-2,19$ \\
\hline Associative activities & 12,14 & $-2,75$ & $-0,81$ & $-2,96$ & 3,61 & $-4,48$ & 5,44 & $-1,51$ & $-2,03$ & 3,24 & $-3,20$ & $-3,85$ & $-7,53$ \\
\hline Rubber-Plastic & 11,99 & 4,80 & 3,75 & 9,84 & 10,73 & 6,96 & 4,49 & 8,59 & 0,67 & 15,70 & 7,49 & 5,83 & 3,79 \\
\hline Education & 11,87 & 11,22 & 5,44 & 6,65 & 8,44 & $-1,68$ & 3,46 & 5,67 & $-0,56$ & $-3,55$ & $-7,71$ & $-2,41$ & $-0,35$ \\
\hline Machines, Apar. Electrical & 11,42 & 13,15 & 19,30 & 11,02 & 12,90 & 4,22 & 13,15 & 12,26 & 14,60 & 10,20 & 13,10 & 7,43 & 7,66 \\
\hline Collection, treatment and distribution & 9,54 & 2,74 & $-7,35$ & 0,23 & 15,11 & 6,05 & 2,28 & 9,16 & $-5,88$ & 18,90 & 7,88 & 12,30 & 2,73 \\
\hline Cellulose-Paper & 9,36 & 0,12 & 8,53 & 0,50 & 1,74 & $-0,03$ & 5,17 & 8,61 & 7,90 & 6,66 & 2,21 & 5,59 & 2,53 \\
\hline Vehicle trade and repair & 9,26 & 5,68 & 2,18 & 2,27 & 4,71 & 1,49 & 2,00 & 5,28 & 9,43 & 8,77 & 5,50 & 6,89 & 2,90 \\
\hline Construction & 6,71 & 0,66 & 1,93 & 2,14 & 4,36 & 3,54 & 4,48 & 6,52 & 14,00 & 16,00 & 16,60 & 18,50 & 14,90 \\
\hline Textiles & 4,52 & $-0,47$ & $-3,49$ & $-7,18$ & $-4,90$ & $-1,18$ & $-5,73$ & $-2,98$ & $-6,21$ & $-4,15$ & $-4,61$ & $-1,41$ & $-4,04$ \\
\hline Recycling & 4,00 & 4,82 & $-6,05$ & $-13,79$ & $-4,96$ & $-11,62$ & $-13,51$ & $-6,69$ & $-2,65$ & $-10,00$ & $-12,00$ & $-2,99$ & $-1,44$ \\
\hline Services provided to companies & 3,05 & $-5,17$ & $-4,97$ & $-3,08$ & $-0,99$ & $-4,40$ & $-3,51$ & $-1,01$ & 0,80 & 2,30 & 0,70 & 0,51 & $-1,57$ \\
\hline Miscellaneous Furniture and Industri & 0,23 & $-5,55$ & $-4,41$ & 0,24 & $-2,93$ & $-1,55$ & $-1,99$ & $-0,83$ & 1,15 & $-3,39$ & 1,07 & 1,74 & $-3,42$ \\
\hline Trade intermediaries & $-0,14$ & $-8,31$ & $-7,98$ & $-5,10$ & $-7,84$ & $-10,17$ & $-8,23$ & $-7,65$ & $-9,15$ & $-8,60$ & $-9,96$ & $-9,87$ & $-13,10$ \\
\hline Agricultural & $-1,28$ & 15,66 & 12,21 & 19,11 & $-15,83$ & 0,09 & 2,89 & $-0,90$ & 13,70 & 30,60 & 27,40 & 44,20 & 29,80 \\
\hline wood & $-2,96$ & $-11,01$ & $-4,82$ & $-7,10$ & $-4,98$ & $-3,40$ & $-3,30$ & $-1,44$ & $-0,41$ & 0,07 & 4,50 & 2,31 & $-1,20$ \\
\hline Real estate activities & $-5,88$ & $-12,84$ & $-10,24$ & $-11,92$ & $-12,32$ & $-13,16$ & $-14,53$ & $-11,13$ & $-4,29$ & $-4,35$ & $-5,69$ & $-4,49$ & $-4,67$ \\
\hline Food-Drinks & $-6,47$ & $-5,55$ & $-8,94$ & $-6,20$ & $-5,10$ & $-8,66$ & $-8,65$ & $-4,32$ & $-6,08$ & $-3,04$ & $-5,61$ & $-2,13$ & $-5,56$ \\
\hline Accommodation and food & $-7,55$ & $-14,25$ & $-14,25$ & $-13,31$ & $-14,03$ & $-13,51$ & $-10,02$ & $-8,67$ & $-12,80$ & $-11,50$ & $-12,10$ & $-11,40$ & $-14,20$ \\
\hline Leathers & $-7,84$ & $-4,86$ & $-9,18$ & $-6,08$ & $-3,01$ & $-9,50$ & $-9,39$ & $-12,19$ & $-15,30$ & $-14,00$ & $-20,60$ & $-18,80$ & $-19,70$ \\
\hline Personal services & $-7,97$ & $-17,49$ & $-4,37$ & $-1,03$ & $-2,81$ & $-3,75$ & $-12,62$ & $-4,40$ & $-9,99$ & $-11,60$ & $-12,40$ & $-5,90$ & $-9,68$ \\
\hline Clothing & $-8,73$ & $-11,13$ & $-12,32$ & $-6,13$ & $-7,52$ & $-9,66$ & $-8,44$ & $-4,61$ & $-16,90$ & $-14,80$ & $-18,80$ & $-16,50$ & $-22,20$ \\
\hline Urban cleaning and sewage & $-20,46$ & $-20,68$ & $-21,16$ & $-11,59$ & $-14,76$ & $-17,48$ & $-18,06$ & $-17,11$ & $-9,98$ & $-11,10$ & $-17,30$ & $-8,74$ & $-9,27$ \\
\hline
\end{tabular}

Source: PNAD/IBGE.

This suggests that workers in these sectors were relatively underpaid for the activities they per- formed. A similar situation was observed among workers in financial institutions, where wages decreased after the inclusion of controls. The uptrend of wage premium in the processing industry is noteworthy, as it occurred during the period in which the sector lost share in value added and productivity dropped.

Firstly, this may be explained by the fact that, despite the lower participation of the sector in the economy, the industry's decline might not have taken place yet and the sector might have kept its more advanced and sophisticated characteristics, where some of the least competitive firms might have taken themselves off the market, leading to a composition effect.

Secondly, there was remarkable growth of production in the industry in the period, but at lower rates than in other sectors, which could have maintained profit in the sector.

Thirdly, the sector might have invested in technologies that require luring and keeping skilled workers, either observable or unobservable human capital.

Fourthly, given the ever-growing lack of skilled workforce and the uncertainties surrounding the future, the 
sector might have retained at least some of the most skilled workers. Also, it is important to consider that low share in the GDP and/or in labor does not always mean that the sector has a lesser importance. More sophisticated indicators, such as industrial density, and international comparisons (Arbache, 2012b) suggest that the industry may influence the paths of the economy even if its direct share in the GDP is not high, as largely witnessed in the USA. While the industry accounts for only $11 \%$ of the value added and $9 \%$ of labor in the USA, it employs $35 \%$ of engineers and $68 \%$ of $\mathrm{R} \& \mathrm{D}$ in the private sector, and accounts for $90 \%$ of patents issued annually and creates, in general, good job opportunities, with wages way above the average and with health insurance and pension plans included (Katz \& Hamp, 2013).

\subsection{Interindustry Wage Premiums}

To analyze the results of wage premiums in more detail, the following sections verify whether the market was more or less segmented, as well as the behavior of the wage structure and the characteristics of the industries that pay higher and lower wages. Controlled and Uncontrolled wage premiums at the one-digit level are reported in Tables 8 and 9, respectively. The coefficients show the proportional difference between the wage of a worker from the agricultural sector and the average weighted wage of a worker from all sectors. In 2015, a worker in the agricultural sector was paid, on average, $24.4 \%$ less than on the market, whereas a worker from the extractive sector earned $33.4 \%$ more. These results might, however, be underestimated or overestimated, as it is reasonable to assume a bias in the selection of workers based on the characteristics of the industry they are affiliated with, and also on factors associated with geographic nd demographic diversity not captured by the data. To minimize the problem, the wage models with individual, geographic, and corporate variables are controlled for. Once controlled, wage premiums change substantially (Table 8). In 2015, the wage in the agricultural sector was $30.6 \%$ lower than that paid on the market. The premium for workers in the extractive sector was $21.5 \%$. There is no single explanation for the change in industrial affiliation coefficients after the control, but selection bias, unmeasured skills and their interaction with industrial affiliation and specification problems are often amongst the major reasons (Gibbons \& Katz, 1992; Arbache, 2001). Results indicate that the wage premium of the extractive sector, which was already high, increased over the decade.

Table 8. Estimated equation with linear restriction for the formal private market with 1 digit and WITHOUT CONTROL for the period 2002-2015

\begin{tabular}{|c|c|c|c|c|c|c|c|c|c|c|c|c|c|}
\hline \multirow{2}{*}{$\begin{array}{l}\text { Variables } \\
\text { Agriculture }\end{array}$} & 02 & 2003 & 2004 & 2005 & 2006 & 2007 & 2008 & 2009 & 2011 & 2012 & 2013 & 2014 & \\
\hline & \multicolumn{13}{|c|}{$-0.327^{a}-0.340^{a}-0.304^{a}-0.283^{a}-0.251^{a}-0.253^{a}-0.225^{a}-0.245^{a}-0.275^{a}-0.139^{a}-0.328^{a}-0.158^{c}-0.244^{a}$} \\
\hline Extra] & & $\begin{array}{l}0.342^{a} \\
(9.82)\end{array}$ & $\begin{array}{l}0.317^{a} \\
(10.4)\end{array}$ & $\begin{array}{l}0.270^{a} \\
(8.89)\end{array}$ & $\begin{array}{l}0.340^{a} \\
(12.3)\end{array}$ & & $\begin{array}{l}0.345^{a} \\
(12.5)\end{array}$ & $\begin{array}{l}0.355^{a} \\
(13.8)\end{array}$ & & & & $\begin{array}{l}0.312^{a} \\
(13.0)\end{array}$ & \\
\hline Tran & & $\begin{array}{l}0.039^{u} \\
(4.00)\end{array}$ & $\begin{array}{l}0.023^{c} \\
(2.52)\end{array}$ & $\begin{array}{l}0.0459^{u} \\
(5.25)\end{array}$ & & & & & & & & & \\
\hline Co & & & & - & & & & & & & & & \\
\hline Cor & & & & & & & & & & & & & \\
\hline & & & & & & & & & & & & & \\
\hline & & & & & & & & & & & & & \\
\hline & & & & & & & & & & & & & \\
\hline & & $\begin{array}{l}0.079^{a} \\
(5.89)\end{array}$ & $\begin{array}{l}0 . \\
(2\end{array}$ & $\begin{array}{l}0 . \\
(2\end{array}$ & $\begin{array}{l}0.0 \\
(2 .\end{array}$ & & & & & & & $\begin{array}{l}0.010^{a} \\
(8.45)\end{array}$ & $\begin{array}{l}0.110^{a} \\
(9.0)\end{array}$ \\
\hline Constant & $3.030^{a}$ & $3.421^{a}$ & $3.713^{a}$ & $4.085^{a}$ & $4.777^{a}$ & $4.501^{a}$ & $\begin{array}{l}4.318^{a} \\
(739)\end{array}$ & $\begin{array}{l}4.711^{a} \\
(862)\end{array}$ & $\begin{array}{l}4.455^{a} \\
(589)\end{array}$ & $4.680^{a}$ & $\begin{array}{l}4.759^{a} \\
(462)\end{array}$ & $\begin{array}{l}4.752^{a} \\
(492)\end{array}$ & $4.328^{a}$ \\
\hline & & & & & & & & & & & 10 & 5935 & 55217 \\
\hline
\end{tabular}

Source: PNAD/IBGE. (t) statistics in parentheses ${ }^{a} p<0.10, \quad{ }^{b} p<0.05, \quad{ }^{c} p<0.01$. 
Table 9. Estimated equation with linear restriction for the formal private market with 1 digit and WITH CONTROL for period 2002-2015

\begin{tabular}{|c|c|c|c|c|c|c|c|c|c|c|c|c|c|}
\hline Variables & 2002 & 2003 & 2004 & 2005 & 2006 & 2007 & 2008 & 2009 & 2011 & 2012 & 2013 & 2014 & 2015 \\
\hline $\begin{array}{l}\text { Grade1 } \\
(\mathrm{t})\end{array}$ & $\begin{array}{l}0,128^{a} \\
(7,13)\end{array}$ & $\begin{array}{l}0,135^{a} \\
(7,20)\end{array}$ & $\begin{array}{l}0,081^{a} \\
(4,23)\end{array}$ & $\begin{array}{l}0,103^{a} \\
(5,64)\end{array}$ & $\begin{array}{l}0,100^{a} \\
(5,29)\end{array}$ & $\begin{array}{l}0,106^{a} \\
(6,32)\end{array}$ & $\begin{array}{l}0,125^{a} \\
(7,77)\end{array}$ & $\begin{array}{l}0,091^{a} \\
(5,31)\end{array}$ & $\begin{array}{l}0,073^{a} \\
(4,46)\end{array}$ & $\begin{array}{l}0,043^{c} \\
(2,42)\end{array}$ & $\begin{array}{l}0,037^{b} \\
(1,81)\end{array}$ & $\begin{array}{l}0,015 \\
(0,83)\end{array}$ & $\begin{array}{l}0,034 \\
(1,52)\end{array}$ \\
\hline Grade2 & $500^{a}$ & $0,482^{a}$ & $0,419^{a}$ & $0,419^{a}$ & $0,393^{a}$ & $0,398^{a}$ & $0,398^{a}$ & $0,348^{a}$ & $0,293^{a}$ & $0,235^{a}$ & $0,217^{a}$ & ${ }^{a} 0,187^{a}$ & $0,196^{a}$ \\
\hline$(\mathrm{t})$ & $(24,7)$ & $(23,6)$ & $(20,3)$ & $(20,9)$ & $(19,4)$ & $(21,5)$ & $(22,7)$ & $(18,9)$ & $(16,5)$ & $(12,3)$ & $(10,1)$ & $(0,08)$ & $(8,19)$ \\
\hline $\begin{array}{l}\text { Undergrad } \\
(\mathrm{t})\end{array}$ & $\begin{array}{l}1,471^{u} \\
(52,9)\end{array}$ & $\begin{array}{l}1,424^{u} \\
(51,0)\end{array}$ & $\begin{array}{l}1,375^{a} \\
(49,7)\end{array}$ & $\begin{array}{l}1,316^{u} \\
(51,2)\end{array}$ & $\begin{array}{l}1,306^{u} \\
(50,6)\end{array}$ & $\begin{array}{l}1,276^{a} \\
(53,9)\end{array}$ & $\begin{array}{l}1,250^{a} \\
(55,0)\end{array}$ & $\begin{array}{l}1,121^{u} \\
(47,8)\end{array}$ & $\begin{array}{l}1,013^{u} \\
(49,7)\end{array}$ & $\begin{array}{l}0,922^{u} \\
(43,5)\end{array}$ & $\begin{array}{l}0,885^{a} \\
(37,3)\end{array}$ & $\begin{array}{l}0,837^{u} \\
(40,9)\end{array}$ & $\begin{array}{l}0,800^{a} \\
(30,9)\end{array}$ \\
\hline Graduate & $2,190^{a}$ & $2,184^{a}$ & $2,011^{a}$ & $2,035^{a}$ & $2,013^{a}$ & $1,957^{a}$ & $2,037^{a}$ & $1,837^{a}$ & $1,817^{a}$ & $1,638^{a}$ & $1,615^{a}$ & $1,616^{a}$ & $1,554^{a}$ \\
\hline$(t)$ & $(26,9)$ & $(28,8)$ & $(28,7)$ & $(30,7)$ & $(30,7)$ & $(32,7)$ & $(39,5)$ & $(30,4)$ & $(33,1)$ & $(33,1)$ & $(33,2)$ & $(32,7)$ & $(28,3)$ \\
\hline exper & $0,034^{a}$ & $0,032^{a}$ & $0,034^{a}$ & $0,028^{a}$ & $0,028^{a}$ & $0,027^{a}$ & $0,024^{a}$ & $0,025^{a}$ & $0,025^{a}$ & $0,019^{a}$ & $0,017^{a}$ & ${ }^{a} 0,018^{a}$ & ${ }^{a} 0,0181^{a}$ \\
\hline (t) & $(24,4)$ & & $(25$ & $(22,0)$ & $(23,5)$ & $(23,4)$ & $(22,4)$ & $(22$, & & 20 & $(17,1)$ & $(2)$ & (18, \\
\hline exper2 & -0 & & & & & & & & & & & & $1 \stackrel{a}{-} 0$, \\
\hline$(\mathrm{t})$ & & & & & & & & & & & & & \\
\hline West & $292^{a}$ & & 0,3 & 0,30 & $0,279^{a}$ & $0,273^{a}$ & $0,267^{a}$ & 0,2 & & 0 & 0,2 & 0 , & ${ }^{a} 0,288^{a}$ \\
\hline$(\mathrm{t})$ & $(19,2)$ & $(21,1)$ & $(21,2)$ & $(22,1)$ & $(22,4)$ & $(21,8)$ & $(22,4)$ & $(20,6)$ & $(19$, & $(23$ & $(19$ & $(26,0)$ & $(24,9)$ \\
\hline South & $0,297^{a}$ & $0,296^{a}$ & $0,291^{a}$ & $0,292^{a}$ & $0,254^{a}$ & $0,244^{a}$ & $0,247^{a}$ & $0,263^{a}$ & $0,174^{a}$ & $0,195^{a}$ & & ${ }^{a} 0,243^{a}$ & ${ }^{a} 0,253^{a}$ \\
\hline$(\mathrm{t})$ & $(22,1)$ & & $(24$, & $(25,3)$ & $(23,8)$ & $(22,8)$ & $(23,6)$ & $(25$ & & $(21$ & $(18$ & $(30$ & $(28,7)$ \\
\hline Southeast & $0,328^{a}$ & $0,342^{a}$ & $0,298^{a}$ & $0,299^{a}$ & $0,271^{a}$ & $0,269^{a}$ & $0,258^{a}$ & $0,244^{a}$ & $0,213^{a}$ & $0,227^{a}$ & $0,190^{a}$ & ${ }^{a} 0,242^{a}$ & ${ }^{a} 0,298^{a}$ \\
\hline (t) & $(29$, & & & & & & $(29,8)$ & $(27,7)$ & $(24,8)$ & $(28,6)$ & & $(33,6)$ & $(36,8)$ \\
\hline North & $0,157^{a}$ & & 0,1 & 0,1 & $0,174^{a}$ & $0,202^{a}$ & $0,143^{a}$ & $0,14^{a}$ & $0,122^{a}$ & $0,151^{a}$ & $0,167^{a}$ & ${ }^{a} 0,194^{a}$ & $0,169^{a}$ \\
\hline$(t)$ & $(9,38)$ & $(10,5)$ & (11 & $(13$, & $(13,6)$ & & & $(12,2)$ & $(10,4)$ & $(13,7)$ & $(13,2)$ & $(18$ & $(15,4)$ \\
\hline Tenure & $0,025^{a}$ & $0,022^{a}$ & $0,022^{a}$ & $0,021^{a}$ & $0,019^{a}$ & $0,018^{a}$ & $0,019^{a}$ & $0,017^{a}$ & $0,128^{a}$ & $0,018^{a}$ & $0,019^{a}$ & $0,018^{a}$ & ${ }^{a} 0,017^{a}$ \\
\hline$(\mathrm{t})$ & $(27,8)$ & $(26,8)$ & $(26$ & $(26,5)$ & $(25,4)$ & $(24,5)$ & $(27,5)$ & $(25,2)$ & $(20,0)$ & $(28,2)$ & $(28,2)$ & $(31,0)$ & $(27,3)$ \\
\hline $\begin{array}{l}\text { Urban } \\
\text { (t) }\end{array}$ & $\begin{array}{l}0,084^{a} \\
(3,68)\end{array}$ & $\begin{array}{l}0,102^{a} \\
(5,82)\end{array}$ & $\begin{array}{l}0,066^{a} \\
(4,07)\end{array}$ & $\begin{array}{l}0,066^{a} \\
(4,62)\end{array}$ & $\begin{array}{l}0,037^{a} \\
(2,82)\end{array}$ & $\begin{array}{l}0,061^{a} \\
(4,66)\end{array}$ & $\begin{array}{l}0,047^{a} \\
(3,52)\end{array}$ & & $\begin{array}{l}0,011 \\
(0,77)\end{array}$ & $\begin{array}{l}0,042^{a} \\
(3,55)\end{array}$ & $\begin{array}{l}0,053^{a} \\
(4,21)\end{array}$ & $\begin{array}{l}0,016 \\
(1,29)\end{array}$ & $\begin{array}{l}0,033^{b} \\
(3,05)\end{array}$ \\
\hline Metrop & $0,085^{a}$ & & $0,050^{a}$ & $0,051^{a}$ & $0,054^{a}$ & $0,040^{a}$ & $0,033^{a}$ & $0,039^{a}$ & $0,089^{a}$ & $0,081^{a}$ & 0,0 & $0,073^{a}$ & $0,111^{a}$ \\
\hline$(t)$ & $(10,2)$ & & & & & & & & & & & & \\
\hline Union & $0,102^{a}$ & $0,101^{a}$ & $0,126^{a}$ & $0,095^{a}$ & $0,096^{a}$ & $0,093^{a}$ & $0,086^{a}$ & $0,080^{a}$ & $0,111^{a}$ & $0,095^{a}$ & $0,105^{a}$ & ${ }^{a} 0,101^{a}$ & ${ }^{a} 0,083^{a}$ \\
\hline$(\mathrm{t})$ & $(10,7)$ & & & $(11,9)$ & $(12,2)$ & $(11,5)$ & $(11,1)$ & $(10,2)$ & $(13,5)$ & $(12,7)$ & $(12,7)$ & $(14,1)$ & $(10,7)$ \\
\hline Npespriv & $0.156^{a}$ & $0.124^{a}$ & $0.130^{a}$ & $0.144^{a}$ & $0.119^{a}$ & $0.114^{a}$ & $0.127^{a}$ & $0.106^{a}$ & $0.064^{a}$ & $0.044^{c}$ & 0.038 & 0.015 & 0.035 \\
\hline$(\mathrm{t})$ & $(15,7)$ & $(13$ & $(14,4)$ & $(16,1)$ & $(14,4)$ & $(14,1)$ & $(16$ & $(13,7)$ & $(15,9)$ & $(14$ & $(13$ & $(16,2)$ & $(13$, \\
\hline White & $0,134^{a}$ & $0,140^{a}$ & $0,135^{a}$ & $0,114^{a}$ & $0,117^{a}$ & $0,116^{a}$ & $0,105^{a}$ & $0,102^{a}$ & $0,105^{a}$ & $0,103 *^{\circ}$ & ${ }^{a} 0,110^{a}$ & ${ }^{2} 0,098^{a}$ & ${ }^{a} 0,100^{a}$ \\
\hline$(\mathrm{t})$ & (1 & $(16,2$ & $(16,4)$ & $(14,7$ & $(16,1)$ & $(15$, & $(14$, & $(14$, & $(15$, & $(16$, & & $(16$ & $(14$, \\
\hline Agriculture & $-0,211$ & $1^{b}$ & $6-0$ & $9=0$ & $9^{\underline{b}}-0$, & $9-0$, & $6-0$, & $0=0$ & $7^{\underline{a}} \mathrm{C}$ & $9^{\underline{\mu}}$ & 2 & $0=0$ & $2^{\underline{a}}-0$, \\
\hline$(t)$ & & & & & & & & & & & & & \\
\hline ExtraIndus & 0,1 & & & 0 , & ses & & & & 0,2 & 0 & $a^{a}$ & $7^{a}$ & ${ }^{a} 0,215^{a}$ \\
\hline$(t$ & $(6$, & & & & & & & & & & & & \\
\hline TransfIndus & $0,029^{b}$ & $0,066^{a}$ & $0,042^{a}$ & $0,052^{a}$ & $0,038^{a}$ & $0,048^{a}$ & $0,047^{a}$ & $0,059^{a}$ & $0,022^{b}$ & 0,0 & 90,0 & 190,0 & $80,0289^{b}$ \\
\hline & $(1,9$ & & & & (0) & & & & & & & $(1$, & $(3,1$ \\
\hline Construc & 0,025 & $0,043^{a}$ & $0,033^{c}$ & $0,027^{c}$ & $0,031^{c}$ & $0,045^{a}$ & $0,046^{a}$ & $0,071^{a}$ & $0,118^{a}$ & $0,142^{a}$ & $0,176^{a}$ & $0,159^{a}$ & $0,166^{a}$ \\
\hline$(t)$ & $(1,49)$ & & & & & & & & & & & & \\
\hline $\begin{array}{c}\text { Commerce } \\
(\mathrm{t})\end{array}$ & $\begin{array}{l}-0,03 \\
(-2,1\end{array}$ & $\begin{array}{l}3 \underline{\underline{b}}-0, \mathrm{c} \\
\text { 4) }(-1,\end{array}$ & 0 & $2^{\underline{a}-0}$ & $\begin{array}{l}7 \underline{\underline{a}}-0, \\
9)(-4\end{array}$ & $8^{\underline{\underline{a}}} 0, \mathrm{C}$ & $9^{\underline{a}-0,}$ & $3^{\underline{a}-0}$, & $9 \underline{a}-0,0$ & $8^{\underline{a}}-0$ & $7^{\underline{a}}-0$ & $5 \stackrel{\underline{a}}{-} 0,100$ & $0^{\underline{a}}-0,085^{c}$ \\
\hline commFood & & & & & & & & & & & & & 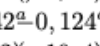 \\
\hline & & & & & & & & & & & & & \\
\hline TranArmCom & $0,089^{a}$ & 0,10 & $0,080^{a}$ & $0,076^{a}$ & $0,053^{a}$ & $0,058^{a}$ & $0,042^{a}$ & $0,069^{a}$ & 0,09 & $0,084^{a}$ & $0,109^{a}$ & $0,074^{a}$ & 0,08 \\
\hline$(\mathrm{t})$ & $(5,34)$ & & & $(4,71)$ & $(3,32)$ & $(4,30)$ & $(3,30)$ & $(5,37)$ & & $(6,88)$ & $(8,49)$ & $(6,53)$ & $(7,45)$ \\
\hline HeathEduc & 0,038 & $0,084^{a}$ & $0,059^{a}$ & 0,032 & 0,031 & 0,015 & 0,007 & $0,049^{c}$ & $0,052^{a}$ & $0,062^{c}$ & $0,067^{a}$ & $0,050^{a}$ & ${ }^{a} 0,013$ \\
\hline$(t)$ & $(1,52)$ & $(3,50)$ & $(2,47)$ & $(1,42)$ & $(1,33)$ & $(0,70)$ & $(0,36)$ & $(2,42)$ & $(4,50)$ & $(4,70)$ & & $(4,06)$ & $(1, \mathrm{C}$ \\
\hline InFinSegPrv & 0,013 & 0,014 & 0,013 & 0,007 & 0,019 & 0,014 & 0,012 & $0,006^{c}$ & $0,022^{c}$ & $0,025^{c}$ & $0,040^{a}$ & 0,005 & $0,033^{b}$ \\
\hline$(\mathrm{t}$ & $(0,78)$ & & & $(0,46)$ & $(1,21)$ & $(1,03)$ & $(0,95)$ & $(0,50)$ & $(2,40)$ & $(2,26)$ & $(3,42)$ & $(0,48)$ & $(3,1$ \\
\hline Constant & $1,549^{a}$ & $1,952^{a}$ & $2,351^{a}$ & $2,783^{a}$ & $3,550^{a}$ & $3,273^{a}$ & $3,122^{a}$ & $3,563^{a}$ & $3,401^{a}$ & $3,676^{a}$ & $3,789^{a}$ & ${ }^{a} 3,823^{a}$ & ${ }^{a} 3,307^{a}$ \\
\hline (t) & $(44,9)$ & $(61$, & $(76,9)$ & & (126) & (126) & (126) & (140) & (143) & (155) & (140) & $(157)$ & (121) \\
\hline Observ. & 23208 & 23498 & 25527 & 27224 & 28251 & 29399 & 30446 & 31494 & 48856 & 51131 & 51705 & 53221 & 49793 \\
\hline
\end{tabular}

Source: PNAD/IBGE. (t) statistics in phßentheses ${ }^{a} p<0.10, \quad{ }^{b} p<0.05, \quad{ }^{c} p<0.01$.

This is likely due, in part, to the substantial increase of commodity prices of ores and petroleum and to the large profits made by firms in this sector. Civil construction, a traditional low-paying sector in Brazil, experienced a hike in wage premium after inclusion of controls in the models. The premium went from $0.5 \%$ in 2015 to $16.8 \%$ after including controls in the model. This upswing apparently mirrors the boom of civil construction in Brazil in the past decade, pushed by credit and large government-subsidized works and their effects on demand for labor, including skilled labor.

\section{Has the Labor Market Become More or Less Segmented?}

If larger integration of the world economy contributes to increasing the market dynamics of wage determination, 
then it would be reasonable to expect convergence of interindustry wage premiums in Brazil and, consequently, a reduction in labor market segmentation. To test this hypothesis, analysis of variance and the coefficient of variation of wage premiums were estimated.

The analysis of variance (Figure 3) shows that the inclusion of controls in the model increases the average coefficient of determination from $8.8 \%$ to $47.6 \%$. Hence, the controls are important to explain wage determination. However, the controls fail to explain wages, as the coefficient of determination decreases monotonically, going from $47.6 \%$ in 2002 to $29.3 \%$ in 2015 . As a matter of fact, the coefficients of the controls shrink over time, including unions, race, geographic regions, schooling, and metropolitan area. Trade union affiliation, for instance, had a $12.1 \%$ impact on wages in 2004, but the coefficient plummeted thereafter, going down to $7.7 \%$ in 2015 .

These results are intriguing. Firstly, they indicate lower wage segmentation associated with controls included in the model. Secondly, the sharper reduction in the coefficient of determination of the model with control indicates relative increase in the importance of industrial affiliation in the share explained in the model. Thus, it is reasonable to assume that industrial affiliation might have become relatively more important to explain wage determination. Thirdly, the decrease in the coefficients of determination suggests greater importance of variables not included in the model in explaining wage dispersion and suggests that wage determination became more complex over the decade.

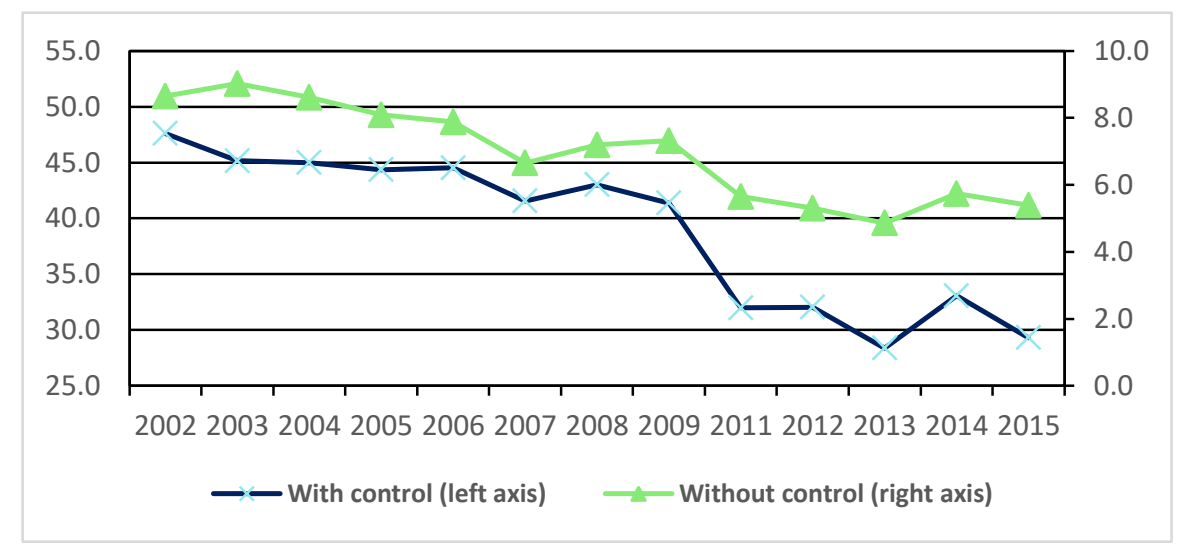

Figure 3. Analysis of variance - 2 digits

Figure 4 shows the coefficient of variation (CV) of wage premiums. The higher the CV, the lower the wage dispersion. The decrease in CV indicates increase in wage dispersion. Dispersion increased in 2002 to 2003 and decreased thereafter until 2015. Therefore, in general, segmentation increased from 2009 onwards, which is likely associated, at least in part, with industrial affiliation and with unmeasured variables.

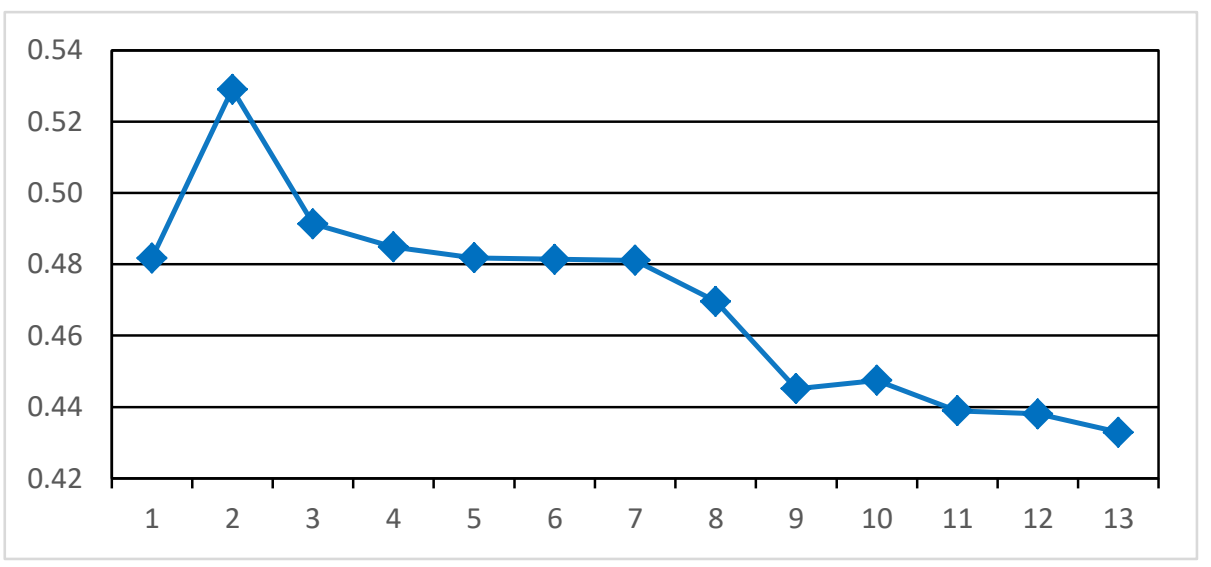

Figure 4. Coefficient of variation, 2 digits with control 


\section{Behavior and Characteristics of the Wage Structure}

\subsection{Changes in Wage Structure}

Table 10 shows that, notwithstanding the changes in labor market, wage structure remained relatively stable from 2002 to 2015 . However, the irregular magnitude of the correlation coefficients and the presence of a statistically nonsignificant coefficient (2013-2014) indicate non-negligible changes in interindustry wage structure (Irregular coefficients are not consistent with similar exercises carried out for Brazil based on comparable data, from the previous period (Arbache, Green, \& Dickerson, 2004a). Although it is reasonable to assert that sectors that paid better (worse) at the beginning of the decade kept playing better (worse) throughout the period, there was a "musical chairs game."

This discrepancy suggests that changes in the wage structure would be associated with the interplay between industrial affiliation, controls, and unmeasured variables.

Table 10. Correlogram of controlled wage premiums at two-digit level

\begin{tabular}{|c|c|c|c|c|c|c|c|c|}
\hline & a2002 & a 2003 & a2004 & a2005 & a2006 & a2007 & a2008 & a2009 \\
\hline a2002 & 1.0000 & & & & & & & \\
\hline a2003 & 0.9192 & 1.0000 & & & & & & \\
\hline a2004 & 0.9216 & 0.9075 & 1.0000 & & & & & \\
\hline a2005 & 0.9365 & 0.9282 & 0.9346 & 1.0000 & & & & \\
\hline a2006 & 0.8401 & 0.9115 & 0.8927 & 0.8781 & 1.0000 & & & \\
\hline a2007 & 0.8865 & 0.9224 & 0.9241 & 0.9252 & 0.9594 & 1.0000 & & \\
\hline a2008 & 0.8593 & 0.8538 & 0.9165 & 0.8703 & 0.9196 & 0.9413 & 1.0000 & \\
\hline a2009 & 0.8561 & 0.8569 & 0.9146 & 0.8770 & 0.9428 & 0.9378 & 0.9415 & 1.0000 \\
\hline a2011 & 0.8302 & 0.7937 & 0.8889 & 0.8447 & 0.8062 & 0.8504 & 0.8806 & 0.9069 \\
\hline a2012 & 0.8779 & 0.8594 & 0.8938 & 0.8917 & 0.8263 & 0.8889 & 0.8428 & 0.8770 \\
\hline a2013 & 0.8963 & 0.8830 & 0.9108 & 0.9039 & 0.9323 & 0.9399 & 0.9254 & 0.9463 \\
\hline a2014 & 0.8788 & 0.8955 & 0.8433 & 0.8988 & 0.8637 & 0.8935 & 0.8247 & 0.8402 \\
\hline \multirow[t]{2}{*}{ a2015 } & 0.8293 & 0.8729 & 0.8922 & 0.8719 & 0.9107 & 0.9271 & 0.8853 & 0.9206 \\
\hline & a2011 & a2012 & a2013 & a2014 & a2015 & & & \\
\hline a2011 & 1.0000 & & & & & & & \\
\hline a2012 & 0.9001 & 1.0000 & & & & & & \\
\hline a2013 & 0.8987 & 0.9099 & 1.0000 & & & & & \\
\hline a2014 & 0.7985 & 0.9067 & 0.8997 & 1.0000 & & & & \\
\hline a2015 & 0.8339 & 0.8232 & 0.9077 & 0.8125 & 1.0000 & & & \\
\hline
\end{tabular}

The 2002 ranking was used as reference. The results confirm the "musical chairs game." The coefficients of the correlogram with uncontrolled premiums (Table 11) are high and stable, which is at odds with the correlogram for controlled premiums (Table 10).

Computer equipment and ancillary financial intermediation activities are among the industries whose relative wages sank, whereas petroleum and alcohol and tobacco are among those industries whose relative wages were pushed up.

Table 11. Correlogram of uncontrolled wage premiums at two-digit level

\begin{tabular}{|c|c|c|c|c|c|c|c|c|}
\hline & a2002 & a2003 & a2004 & a2005 & a2006 & a2007 & a2008 & a2009 \\
\hline a2002 & 1.0000 & & & & & & & \\
\hline a2003 & 0.9947 & 1.0000 & & & & & & \\
\hline a2004 & 0.9932 & 0.9974 & 1.0000 & & & & & \\
\hline a2005 & 0.9919 & 0.9971 & 0.9993 & 1.0000 & & & & \\
\hline a2006 & 0.9691 & 0.9876 & 0.9858 & 0.9866 & 1.0000 & & & \\
\hline a2007 & 0.9820 & 0.9933 & 0.9947 & 0.9945 & 0.9958 & 1.0000 & & \\
\hline a2008 & 0.9663 & 0.9844 & 0.9846 & 0.9850 & 0.9969 & 0.9969 & 1.0000 & \\
\hline a2009 & 0.9593 & 0.9805 & 0.9790 & 0.9795 & 0.9989 & 0.9928 & 0.9969 & 1.0000 \\
\hline a2011 & 0.9659 & 0.9820 & 0.9811 & 0.9819 & 0.9956 & 0.9945 & 0.9978 & 0.9964 \\
\hline a2012 & 0.9514 & 0.9708 & 0.9684 & 0.9689 & 0.9917 & 0.9880 & 0.9950 & 0.9948 \\
\hline a2013 & 0.9423 & 0.9622 & 0.9607 & 0.9601 & 0.9871 & 0.9828 & 0.9909 & 0.9917 \\
\hline a2014 & 0.9383 & 0.9596 & 0.9605 & 0.9610 & 0.9867 & 0.9835 & 0.9922 & 0.9912 \\
\hline \multirow[t]{2}{*}{ a2015 } & 0.9474 & 0.9648 & 0.9668 & 0.9676 & 0.9864 & 0.9862 & 0.9928 & 0.9900 \\
\hline & a2011 & a2012 & a2013 & a2014 & a2015 & & & \\
\hline a2011 & 1.0000 & & & & & & & \\
\hline a2012 & 0.9978 & 1.0000 & & & & & & \\
\hline a2013 & 0.9947 & 0.9987 & 1.0000 & & & & & \\
\hline a2014 & 0.9939 & 0.9979 & 0.9980 & 1.0000 & & & & \\
\hline a2015 & 0.9960 & 0.9975 & 0.9965 & 0.9984 & 1.0000 & & & \\
\hline
\end{tabular}


To assess wage structure, we decomposed the within-group and between-group components of wage premium variancen (Wage premium variance can be decomposed into premium variance within each industry over time (within-group component) and premium variance between industries over time (between-group component). If the wage structure is actually very strict, then, ceteris paribus, one should expect the between-group component to be similar to or lower than the within-group component. However, if the wage structure experienced remarkable changes, one should expect the between-group component to be much higher than the within-group component. The within-group component was 8.01\%, whereas the between-group component was $14.71 \%$, nearly twice as high, corroborating important changes in the wage structure.

Table 12. Ranking salary premium - 2 digits - with control

\begin{tabular}{|c|c|c|c|c|c|c|c|c|c|c|c|c|c|}
\hline Industries & 2002 & 2003 & 2004 & 2005 & 2006 & 2007 & 2008 & 2009 & 2011 & 2012 & 2013 & 2014 & 2015 \\
\hline Air Transport & 1 & 2 & 4 & 1 & 2 & 2 & 5 & 2 & 3 & 3 & 2 & 4 & 3 \\
\hline Oil extraction & 2 & 1 & 2 & 2 & 3 & 1 & 1 & 1 & 1 & 1 & 1 & 1 & 1 \\
\hline Equip. IT & 3 & 3 & 24 & 18 & 10 & 9 & 3 & 16 & 24 & 24 & 17 & 6 & 36 \\
\hline Aux. Activities interm. financial & 4 & 25 & 9 & 7 & 26 & 17 & 6 & 14 & 29 & 29 & 22 & 24 & 20 \\
\hline Interm. Financ.seg. and previd. Priv. & 5 & 4 & 8 & 5 & 7 & 4 & 7 & 5 & 9 & 9 & 10 & 10 & 6 \\
\hline Computer activities & 6 & 5 & 5 & 4 & 14 & 8 & 9 & 6 & 6 & 6 & 8 & 9 & 9 \\
\hline Equip. Transport & 7 & 16 & 16 & 15 & 9 & 13 & 10 & 11 & 13 & 13 & 5 & 5 & 14 \\
\hline Equip. from instrum.hospitalares & 8 & 19 & 13 & 20 & 13 & 22 & 17 & 10 & 20 & 20 & 26 & 26 & 25 \\
\hline Research and Development & 9 & 22 & 1 & 43 & 47 & 21 & 44 & 37 & 5 & 5 & 28 & 22 & 32 \\
\hline Electricity, gas and hot water & 10 & 8 & 17 & 19 & 11 & 12 & 16 & 7 & 10 & 10 & 6 & 12 & 7 \\
\hline Apar.Equip. Communications & 11 & 13 & 14 & 15 & 16 & 15 & 21 & 24 & 32 & 32 & 14 & 13 & 46 \\
\hline Auto-vehicles & 12 & 7 & 7 & 8 & 8 & 5 & 12 & 12 & 11 & 11 & 12 & 14 & 13 \\
\hline Extraction of metallic minerals & 13 & 9 & 6 & 13 & 5 & 6 & 4 & 5 & 4 & 4 & 3 & 2 & 12 \\
\hline Chemicals & 14 & 20 & 20 & 17 & 15 & 18 & 18 & 20 & 16 & 16 & 21 & 23 & 18 \\
\hline Edition-Print & 15 & 10 & 29 & 22 & 28 & 24 & 24 & 26 & 25 & 25 & 35 & 18 & 23 \\
\hline Car rental, machine. and equip. & 16 & 17 & 42 & 27 & 32 & 20 & 22 & 38 & 34 & 34 & 18 & 45 & 22 \\
\hline Water transportation & 17 & 23 & 11 & 14 & 6 & 10 & 23 & 4 & 2 & 2 & 4 & 8 & 3 \\
\hline Basic Metallurgy & 18 & 18 & 16 & 21 & 18 & 14 & 16 & 17 & 14 & 14 & 11 & 16 & 11 \\
\hline Machines-Equipment & 19 & 11 & 18 & 11 & 12 & 11 & 14 & 18 & 22 & 22 & 15 & 19 & 15 \\
\hline Metal Products & 20 & 28 & 22 & 26 & 23 & 19 & 20 & 23 & 18 & 18 & 24 & 20 & 19 \\
\hline Smoke & 21 & 30 & 10 & 29 & 4 & 3 & 8 & 9 & 7 & 7 & 13 & 47 & 10 \\
\hline Extraction of mineral coal & 22 & 12 & 3 & 3 & 1 & 53 & 2 & 15 & 15 & 15 & 9 & 11 & 2 \\
\hline Insurance and private pension & 23 & 6 & 37 & 10 & 19 & 23 & 13 & 19 & 8 & 8 & 25 & 28 & 26 \\
\hline Non-Metallic Minerals & 24 & 27 & 34 & 40 & 37 & 40 & 26 & 21 & 26 & 26 & 36 & 30 & 44 \\
\hline Post and telecommunications & 25 & 14 & 19 & 23 & 24 & 25 & 27 & 28 & 31 & 31 & 34 & 38 & 38 \\
\hline Activ. aux. of transp. ag. on a trip & 26 & 41 & 26 & 33 & 33 & 26 & 35 & 33 & 30 & 30 & 33 & 35 & 21 \\
\hline Oil-Alcohol & 27 & 26 & 23 & 12 & 17 & 7 & 11 & 8 & 12 & 12 & 16 & 7 & 8 \\
\hline Recreational, cultural activities & 28 & 37 & 32 & 32 & 29 & 30 & 28 & 36 & 35 & 35 & 37 & 27 & 40 \\
\hline Extraction of non-metallic minerals & 29 & 35 & 28 & 9 & 25 & 16 & 25 & 13 & 17 & 17 & 19 & 15 & 17 \\
\hline Transport, Warehousing and Commun & 30 & 29 & 30 & 30 & 31 & 31 & 33 & 34 & 27 & 27 & 27 & 25 & 27 \\
\hline Health and social services & 31 & 31 & 25 & 31 & 30 & 33 & 34 & 25 & 42 & 42 & 42 & 38 & 39 \\
\hline Associative activities & 32 & 42 & 38 & 41 & 36 & 44 & 29 & 43 & 41 & 41 & 41 & 44 & 47 \\
\hline Rubber-Plastic & 33 & 34 & 33 & 25 & 22 & 27 & 31 & 30 & 38 & 38 & 30 & 32 & 28 \\
\hline Education & 34 & 24 & 31 & 28 & 27 & 39 & 36 & 32 & 40 & 40 & 46 & 42 & 33 \\
\hline Machines, Apar. Electrical & 35 & 21 & 12 & 24 & 21 & 29 & 19 & 22 & 19 & 19 & 23 & 23 & 24 \\
\hline Collection, treatment and distribution. Water & 36 & 36 & 46 & 38 & 20 & 28 & 38 & 27 & 45 & 45 & 29 & 21 & 30 \\
\hline Cellulose-Paper & 37 & 39 & 27 & 36 & 38 & 36 & 30 & 29 & 33 & 33 & 38 & 23 & 31 \\
\hline Vehicle trade and repair & 38 & 32 & 35 & 34 & 34 & 34 & 39 & 35 & 28 & 28 & 32 & 31 & 29 \\
\hline Construction & 39 & 38 & 36 & 35 & 34 & 34 & 39 & 35 & 21 & 21 & 20 & 17 & 16 \\
\hline Textiles & 40 & 40 & 39 & 49 & 43 & 37 & 43 & 44 & 47 & 47 & 43 & 40 & 42 \\
\hline Recycling & 41 & 33 & 45 & 53 & 44 & 49 & 51 & 48 & 43 & 43 & 48 & 43 & 35 \\
\hline Services provided to companies & 42 & 44 & 44 & 42 & 39 & 43 & 42 & 41 & 37 & 37 & 40 & 39 & 37 \\
\hline Miscellaneous Furniture and Industries & 43 & 45 & 41 & 37 & 41 & 38 & 40 & 39 & 36 & 36 & 39 & 37 & 41 \\
\hline Trade intermediaries & 44 & 47 & 47 & 44 & 49 & 48 & 45 & 49 & 48 & 48 & 47 & 50 & 50 \\
\hline Agricultural & 45 & 15 & 21 & 16 & 53 & 35 & 37 & 40 & 23 & 23 & 7 & 3 & 4 \\
\hline wood & 46 & 48 & 43 & 48 & 45 & 41 & 41 & 42 & 39 & 39 & 34 & 36 & 34 \\
\hline Real estate activities & 47 & 50 & 50 & 51 & 50 & 50 & 52 & 51 & 44 & 44 & 45 & 45 & 43 \\
\hline Food-Drinks & 48 & 46 & 48 & 47 & 46 & 45 & 47 & 45 & 45 & 46 & 44 & 41 & 46 \\
\hline Accommodation and food & 49 & 51 & 52 & 52 & 51 & 51 & 49 & 50 & 51 & 51 & 49 & 51 & 51 \\
\hline Leathers & 50 & 43 & 49 & 45 & 42 & 46 & 48 & 52 & 52 & 53 & 53 & 53 & 52 \\
\hline Personal services & 51 & 52 & 40 & 39 & 40 & 42 & 50 & 46 & 50 & 50 & 50 & 48 & 49 \\
\hline Clothing & 52 & 49 & 51 & 46 & 48 & 47 & 46 & 47 & 53 & 53 & 52 & 52 & 53 \\
\hline Urban cleaning and sewage & 53 & 53 & 53 & 50 & 52 & 52 & 53 & 53 & 49 & 51 & 51 & 49 & 48 \\
\hline
\end{tabular}

Source: PNAD/IBGE.

\section{Characteristics of Sectors That Pay Better and Worse}

Table 13 shows the 12 sectors with the highest and lowest wage premiums. Commodities sectors and those sectors with highly concentrated markets, such as air transport, petroleum extraction, mineral coal extraction, financial institutions, and automotive industry, and more technology-intensive sectors are among those which 
pay the highest premiums. On the other hand, sectors with more competitive markets, such as clothing, foods, recycling, and trade, sectors that use less technology, such as urban cleaning, housing, and wood, and sectors that require less capital stock are among those which pay the lowest premiums

Table 13. 12 Minors and largest averages salary premiums average values

\begin{tabular}{lclc}
\hline Smallest & Averages & Larger & Averages \\
\hline Têxteis & $-15,21$ & Oil extraction & 58,28 \\
Food-Drinks & $-12,13$ & Air Transport & 47,84 \\
Recycling & $-12,12$ & Extraction of mineral coal & 39,43 \\
Personal services & $-11,57$ & Extraction of metal minerals & 33,64 \\
Trade intermediaries & $-8,89$ & Interm. Fin.seg.previd. Priv. & 28,73 \\
Real estate activities & $-8,16$ & Water transportation & 27,80 \\
Leathers & $-8,00$ & Computer activities & 27,48 \\
Accommodation-ood & $-5,91$ & Auto-vehicles & 25,28 \\
Clothing & $-5,87$ & Equip. Transport & 24,81 \\
Urban cleaning-ewage & $-3,22$ & Equip. IT & 23,54 \\
Wood & $-2,60$ & Electricity, gas-hot water & 22,88 \\
Miscell. Furniture. ndustries & $-1,51$ & Oil-Alcohol & 21,79 \\
\hline
\end{tabular}

Source: PNAD/IBGE.

Table 14 compares the 2002 and 2015 rankings and shows the sectors whose premiums increased and decreased the most. Among the sectors whose premiums increased the most are those directly or indirectly linked to the primary sector, such as mineral extraction, mineral coal, non-metallic minerals, petroleum and alcohol, and tobacco. Among the sectors whose premiums decreased the most are those industries with various characteristics, including sectors that experienced substantial increase in international competition during the period. Negative and statistically significant correlation between wage premium and labor force participation suggests that better-paying sectors have fewer job openings and vice versa. In fact, the sectors that pay the lowest 12 premiums employ $39.6 \%$ of workers, whereas sectors that pay the highest premiums employ $5.9 \%$ of workers.

Table 14. Most significant changes comparing the 2002 and 2015 rankings

\begin{tabular}{lccc}
\hline Industries & $\mathbf{2 0 0 2}$ & $\mathbf{2 0 1 5}$ & Movement \\
\hline Water transportation & 17 & 5 & Elevation \\
Tobacco Industry & 21 & 10 & Elevation \\
Extraction of mineral coal & 22 & 2 & Elevation \\
Oil-Alcohol & 27 & 8 & Elevation \\
Extraction of non-metallic minerals & 29 & 17 & Elevation \\
Construction & 39 & 16 & Elevation \\
Agricultural & 46 & 4 & Elevation \\
Equip. IT & 3 & 36 & Fall \\
Aux. Activities interm. financial & 4 & 20 & Fall \\
Equip. Transport & 7 & 14 & Fall \\
Equip. from instrum.hospitalares & 8 & 25 & Fall \\
Research and Development & 9 & 32 & Fall \\
Apar.Equip. Communications & 11 & 46 & Fall \\
Edition-Print & 15 & 23 & Fall \\
Non-Metallic Minerals & 24 & 44 & Fall \\
\hline
\end{tabular}

Source: PNAD/IBGE.

\section{Wage Premium and Competition}

This section assesses the prospects of cross-sector competition and welfare based on the behavior and dynamics of interindustry wage premium. The goal is to identify sectors that simultaneously experience an increase in premium and in job creation.

Three sectors unequivocally meet the established criteria: petroleum and alcohol, extraction of metallic minerals, 
and civil construction. Of these three sectors, two in the commodities market, prices increased in the period thanks to the favorable economic scenario in Brazil and to the boost in demand from China. In the case of petroleum, the sector benefited from discoveries of, and investments in, pre-salt. The tertiary sector, civil construction, is concerned with essentially domestic activities that benefited from public policies. Informatics activities also fall under this metric, but only from the mid-2000s onwards.

Although these sectors have offered more job openings, the petroleum and alcohol, and metallic minerals sectors employ far fewer workers in relative terms - in 2015, they accounted for a little bit more than $1 \%$ of total labor (Table 4). The petroleum sector is, however, extremely prosperous, because oil reserves in the pre-salt layer are subject to several restrictions that prevent its extraction by the available technologies. Moreover, the distance between the coast and the future oil wells increases logistics complexity. To circumvent these problems, tens of billion dollars would have to be invested in new technologies. Since technologies are not properly mastered yet, there are a huge variety of opportunities for investing in knowledge and industrial advances. If developed by universities and research centers in Brazil and incorporated by the industry, this knowledge and these competencies may have profound impact on several other industrial sectors, with unprecedented economic and social effects. This frontier of development shows great potential for the creation of high-quality jobs, static and dynamic increasing returns, externalities, productivity gains, high value added, and development of skills and competencies. The presence of a positive effect of firm size on workers' wages is well documented in the economic literature (Oi \& Idson, 1999a). Edin and Zetterberg (1992) and Arai (1994) find considerable differences in wage levels between industries when controlling for the characteristics of individuals and firms.

Furthermore, this is the biggest opportunity known so far for investments, consolidation, and stimulation of production chains in Brazil. Pre-salt should therefore be viewed from the perspective of strategic economic policy.

The mineral extraction sector, in turn, has a much shorter production chain and fewer chances of improving Brazil's systemic productivity and competitiveness. Its most visible positive impact lies in the balance of trade. No matter how much the sector grows, it will not be an essential integral part of the equation for the increase in competitiveness and welfare, unless it is associated with specific fiscal policies and value-added industrial policies.

Civil construction accounts for a significant share of labor $(6.25 \%)$, outranked only by trade $(10.1 \%)$ and agriculture $(6.54 \%)$. Given the large housing deficit in Brazil, the big infrastructure challenges, and the long production chain of this sector, the growth of civil construction may have significant impact on income and labor, especially if followed by an increase in technological knowledge, innovation, and labor productivity.

Some sectors do not meet the criteria established herein, but their wage premiums are high and employ a relatively large share of workers. According to this metric, the following sectors were identified: transport and storage, trade, and vehicle, machinery, and equipment repair.

\section{Conclusions}

This paper revisits interindustry wage differential in Brazil in a period of quick and intense labor market and output changes. The major conclusions of this study are described in what follows. First, the labor market was more segmented and more complex. On the one hand, there was a decrease in wage dispersion associated with variables that are typically used in studies of this nature. On the other hand, there was a relative increase in the importance of industrial affiliation and a significant increase in the importance of variables not measured by conventional statistics to explain wage dispersion. Owing to the larger integration of the Brazilian economy with the international economy, these results are in line with the expectations, suggesting that the labor market may have gone through extensive changes.

Secondly, there were changes in wage structure. Relative wages increased in some sectors but decreased in others. These changes suggest a tendency towards the reallocation of resources at the sectoral level.

As the dynamics of interindustry wage premium is associated with competitive potential, our analysis may be useful for the formulation of industrial, technological, innovation, human capital, foreign trade, labor, and income policies. The transfer of funds to sectors whose premiums show a tendency towards growth would increase, ceteris paribus, good-quality jobs, productivity, and value added.

The petroleum and alcohol, mineral extraction, and civil construction sectors have a large potential for increase in value added and labor. However, if indirect effects are taken into account, notably through value chains, the petroleum and alcohol and civil construction sectors would make major contributions.

Sectors such as transport and storage, trade, and vehicle, machinery, and equipment repair have potential, albeit more moderate, for an increase in income and value added due to the high level of wage premium and of labor. 


\section{References}

Aghion, P., Dewatripont, M., Du, L., Harrison, A. E., \& Legros, P. (2011). Industrial policy and competition. SSRN \#1811643. https://doi.org/10.2139/ssrn.1811643

Allen, S. (1995). Update Notes on the Inter-industry Wage Structure: 1890-1990. Industrial and Labor Relations Review, 48, 305-21. https://doi.org/10.1177/001979399504800207

Arai, M. (1994). An Empirical Analysis of Wage Dispersion and Efficiency Wages. Scandinavian Journal of Economics, 96, 31-50. https://doi.org/10.2307/3440665

Arbache, J. (2001). Wage differentials in Brazil: Theory and evidence. Journal of Development Studies, 38, 109-130. https://doi.org/10.1080/00220380412331322281

Arbache, J. (2012a). Transformação demográfica e competitividade internacional da economia brasileira. Revista do BNDES, 36, 365-392.

Arbache, J. (2012b). Is Brazilian manufacturing losing its drive? SSRN \#2150684. https://doi.org/10.2139/ssrn.2150684

Arbache, J., \& Corseuil, C. H. (2004). Liberalização comercial e estruturas de emprego e salários. Revista Brasileira de Economia, 58, 485-505. https://doi.org/10.1590/S0034-71402004000400002

Arbache, J., \& De Negri, J. A. (2004). Diferenciais de salários interindustriais no Brasil: Evidências e $\begin{array}{lllll}\text { implicações. Revista Brasileira de } & \text { Economia, } & 58, & 159-184 .\end{array}$ https://doi.org/10.1590/S0034-71402004000200002

Arbache, J., Dickerson, A., \& Green, F. (2001), A picture of wage inequality and the allocation of labor through a period of trade liberalization: The case of Brazil. World Development, 29, 1923-1939. https://doi.org/10.1016/S0305-750X(01)00071-7

Arbache, J., Dickerson, A., \& Green, F. (2004a). Assessing the Stability of the Inter-industry Wage Structure in the Face of Radical Economic Reforms. Economics Letters, 83, 149-155. https://doi.org/10.1016/j.econlet.2003.10.013

Arbache, J., Dickerson, A., \& Green, F. (2004b). Trade liberalization and wages in developing countries. Economic Journal, 114, 73-96. https://doi.org/10.1111/j.0013-0133.2004.00188.x

Brander, J. (1995). Strategic trade policy. NBER WP 5020.https://doi.org/10.3386/w5020

Dickens, W. T., \& Katz, L. F. (1987). Inter-industry Wage Differences and Industry Characteristics. In K. Lang, \& J. Leonard (Eds.), Unemployment and the Structure of Labor Markets. Oxford: Basil Blackwell. https://doi.org/10.3386/w2271

Doeringer, P. B., \& Piore, M. J. (1971). Internal labor markets and manpower analysis. Lexington: D.C. Health $\&$ Co.

Eaton, J., \& Grossman, G. N. (1986). Optimal trade and industrial policy under oligopoly. Quarterly Journal of Economics, 101, 383-406. https://doi.org/10.2307/1891121

Gibbons, R., \& Katz, L. (1992). Does Unmeasured Ability Explain Inter-Industry Wage Differen- tials? Review of Economic Studies, 59, 515-535. https://doi.org/10.2307/2297862

Haisken-DeNew, J. P., \& Schmidt, C. M. (1997). Inter-Industry and Inter-Region Differentials: Mechanics and Interpretation. Review of Economics and Statistics, 79, 516-521. https://doi.org/10.1162/rest.1997.79.3.516

IPEA. (2012). Produtividade no Brasil nos anos 2000-2009: Análise das Contas Nacionais. Comuni- cados IPEA No. 133.

Jayanthakumaran, K., Piyapong, S., \& Martin, O. (2013). Trade liberalisation and manufacturing wage premiums: Evidence from Thailand. Journal of Asian Economics, 29, 15-23. https://doi.org/10.1016/j.asieco.2013.08.001

Katz, B., \& Hamp, P. (2013). The U.S. must 'race to the shop' to spur economic growth. Brookings, Series: Remaking Federalism, \#21.

Katz, L. F., \& Summers, L. H. (1989). Industry Rents: Evidence and Implications. Brookings Papers on Economic Activity, Microeconomics, 209-275. https://doi.org/10.2307/2534722

Krueger, A. B., \& Summers, L. H. (1987). Reflections on Inter-Industry Wage Structure. In K. Lang, \& J. Leonard (Eds.), Unemployment and the Structure of Labor Markets. Oxford: Basil Blackwell. 
https://doi.org/10.3386/w1968

Krueger, A. B., \& Summers, L. H. (1988). Efficiency Wages and The Inter-Industry Wage Structure. Econometrica, 56, 259-293. https://doi.org/10.2307/1911072

Murphy, K. M., \& Topel, R. H. (1990). Efficiency Wages Reconsidered: Theory and Evidence. In Y. Weiss, \& G. Fishelson (Eds.), Advances in Theory and Measurement of Unemployment. London: Macmillan. https://doi.org/10.1007/978-1-349-10688-2_8

Nuun, N., \& Trefler, D. (2010). The structure of tariffs and long-term growth. American Economic Journal: Macroeconomics, 2, 158-194. https://doi.org/10.1257/mac.2.4.158

Oi, W. Y., \& Idson, T. L. (1999a). Firm Size and Wages. In O. Ashenfelter, \& D. Card (Eds.), Handbook of labor economics (3rd ed.). Amsterdam: North-Holland,

Per-Anders, E., \& Johnny, Z. (1992). Interindustry Wage Differentials: Evidence from Sweden and a Comparison with the United States. American Economic Review, 82, 1341-49.

Spencer, B., \& Brander, J. A. (2008). Strategic trade policy. In S. N. Durlauf, \& L. E. Blume (Eds.), The New Palgrave Dictionary of Economics. Palgrave Macmillan. https://doi.org/10.1057/978-1-349-95121-5_2264-1

Todd, L. I., \& Walter, Y. O. (1999b). Workers Are More Productive in Large Firms. The American Economic Review, 89(2). Papers and Proceedings of the One Hundred Eleventh Annual Meeting of the American Economic Association (May, 1999), pp. 104-108. https://doi.org/10.1257/aer.89.2.104

\section{Copyrights}

Copyright for this article is retained by the author(s), with first publication rights granted to the journal.

This is an open-access article distributed under the terms and conditions of the Creative Commons Attribution license (http://creativecommons.org/licenses/by/4.0/). 